\title{
Measuring Higher-Mode Surface Wave Dispersion Using a Transdimensional Bayesian Approach
}

\author{
H. $\mathrm{Xu}^{1}$, C. Beghein ${ }^{1,2}$ \\ ${ }^{1}$ Department of Earth, Planetary, and Space Sciences, University of California Los Angeles, Los Angeles, \\ CA 90095, USA \\ ${ }^{2}$ Lunar and Planetary Institute, 3600 Bay Area Blvd, Houston, TX 77058, USA
}

\section{SUMMARY}

We present a method for measuring the dispersion of higher mode surface wave phase velocities from a single seismogram using a hierarchical transdimensional Bayesian approach. The 1-D shear velocity profiles down to $800 \mathrm{~km}$ depth between sources and seismic stations are regarded as the controlling parameters to tune the phase velocities of fundamental and higher modes. The misfits between synthetics and real waveforms indicate whether the phase velocities are recovered well from the data. We use Monte Carlo Markov chains (MCMC) to approximate the posterior distribution of each model parameters, and assess the uncertainties from these probability density functions. These techniques can test models of varying dimensions while being parsimonious, thereby letting the data themselves control the complexity of the solution. Another advantage is that the algorithm can decide how much data noise is needed in order to explain the data without overfitting them. The data noise can be treated as an unknown and different noise levels can be applied to the different time windows considered. The posterior noise distributions can then be used as an indicator of the quality of the waveform fit within each frequency-time window. We considered phase velocities between 50s and 200s for each 
mode, and performed a reliability analysis to determine which modes and periods are reliably constrained. In this paper, we first present the method and demonstrate its feasibility with synthetic tests, which show that the technique is robust. We then illustrate it with applications to real data. We applied the method to two paths sampling Australia using earthquakes at regional distances, and obtained results that agree well with previous studies. The new method can be used in regional and global tomographic studies to obtain phase velocity maps and 3-D models of seismic velocities and anisotropy at depths that are not well resolved by fundamental mode surface waves or body waves.

Key words: Surface waves and free oscillations - Probability distributions - Tomography - Inverse theory - Statistical seismology.

\section{INTRODUCTION}

Higher mode surface waves carry unique, independent constraints on structure at greater depths than commonly used fundamental mode surface waves, and thus enhance the vertical resolution of surface wave tomographic models in the deep upper mantle, transition zone, and uppermost lower mantle. However, most surface wave tomography studies are still limited to fundamental surface waves because traditional surface wave measurements can only be readily applied to fundamental modes and measuring higher modes is complicated. Only in very specific and rare cases has it been possible to isolate and measure the dispersion of the first higher mode Rayleigh wave using single mode dispersion methods (Crampin, 1964, Roult and Romanowicz, 1984). The difficulties in measuring higher modes are mainly due to the fact that their group velocities overlap significantly in a broad frequency range and thus they do not appear as a clear wave train on the seismogram. When distant events are used, a small difference in group velocity can be sufficient to obtain mode separation using numerical techniques, but it is not always enough.

One way to take advantage of the information contained in higher-mode surface waves is to retrieve velocity structure directly, without extracting dispersion curves first. Most of these techniques involve determining path-averaged shear wave velocity structure for multiple station-receiver pairs and combining them to obtain 3-D models of Earth's internal structure. Cara and Lévêque (1987) determined path-averaged $V_{S}$ models from a single seismogram using mode branch cross-correlation functions (Lerner-Lam and Jordan, 1983) as secondary observables. This method, which was designed to minimize the dependence on the starting model, was later automated and combined with a regionalization scheme (Montagner, 1986) to model 3-D velocities (Debayle, 1999) and anisotropy (Maggi et 
al., 2006). Nolet (1990) developed a two-step non-linear Partitioned Waveform Inversion (PWI) technique that was automated by Lebedev and Nolet (2003). Using synthetic seismogram calculations, the PWI finds along-path velocity models that fit recorded waveforms and inverts the velocity models obtained for all paths to constrain 3-D structure. Li and Romanowicz (1995; 1996) were also able to obtain the averaged structure along the great circle between the source and receiver using a waveform modeling technique that included non-linear asymptotic coupling theory and coupling across different branches.

Despite the difficulty, several attempts have been made to separate higher modes. Some methods operate in the frequency-wavenumber $(\omega-k)$ domain and use seismic arrays (Nolet, 1975; Cara, 1973; Cara, 1978: Cara, 1979). While this kind of direct measurement is fast to implement, there are a few limitations: (i) the method can only be applied in regions with dense arrays and thus cannot be applied at the global scale, (ii) it requires the usage of linear regional seismic arrays approximately aligned with the epicenter, which reduces the geographical ray coverage, and (iii) variations within an array cannot be assessed (Laske and Widmer-Shnidrig, 2015).

Another method, based on mode branch stripping, was developed by van Heijst and Woodhouse (1997). The principle behind this technique is that the signals of overtone branches can be isolated by fitting the cross-correlation function of a single mode using mode branch cross-correlation functions. However, while this method does not rely on arrays and thus can be used in global tomography, it does not work well with epicentral distances shorter than $30^{\circ}$ (van Heijst and Woodhouse, 1999) since individual higher modes cannot be separated from others in a seismogram when the path is too short. Additionally, it might be difficult to separate one single mode from observed seismograms when that mode is contaminated by interference from other modes.

Other researchers have utilized waveform fitting techniques to extract higher-mode dispersion. Stutzmann and Montagner (1993) and Stutzmann et al. (1994) developed a waveform fitting technique to invert phase velocity dispersion and velocity structure at depth in successive steps. Beucler et al. (2003) proposed a roller-coaster technique, in which the phase velocity perturbation is obtained by fitting the synthetic seismogram to the real data. Yoshizawa and Kennett (2002, 2004) and Yoshizawa and Ekström (2010) used the Neighborhood Algorithm (Sambridge, 1999a) to search the model space for multimode dispersion in a nonlinear waveform inversion. Visser et al. (2007) developed a method similar to that of Yoshizawa and Kennett (2002) to estimate multimode dispersion curves for global tomography, though they were additionally able to obtain quantitative uncertainties on the phase speed measurements by performing a Bayesian appraisal of the models sampled (Sambridge, 1999b). Uncertainties were determined from the whole ensemble of dispersion models obtained instead of taking the standard deviation of the 1,000 best models as in Yoshizawa and Kennett (2002). 
In our study, we developed a waveform modeling method using a reversible jump Markov Chain Monte Carlo (rj-MCMC) inversion (Bodin and Sambridge, 2009) to extract higher mode dispersion curves and their uncertainties at periods between 50s and 200s. Like the methods of Yoshizawa and Kennett (2002), Yoshizawa and Ekström (2010), and Visser et al. (2007; 2008), our technique has the advantage of sampling the model space instead of choosing one model among many possible solutions with a strong regularization scheme. It therefore provides more reliable posterior model uncertainties than regularized inversions. Additionally, contrary to those previous studies in which the authors adopted a fixed dimension model space chosen a priori, the rj-MCMC sampler offers a way to treat the dimension of the model space as variable. In addition, because we use a Hierarchical Bayes approach, data noise parameters are treated as unknowns in the inversion, which accounts for the part of the signal that we are not able to explain with our forward theory and the chosen parametrization. This allows the model to explain the data without overfitting them. In this paper, we first present the method and validate it using a synthetic test, and then show two real data examples.

\section{METHOD}

Our goal is to measure phase velocity dispersion for fundamental and higher mode Rayleigh waves using waveform modeling. To do this, we seek a large number of path-averaged 1-D shear-velocity models that can fit the filtered waveform, and the resulting models are employed to calculate dispersion curves. These path-specific 1-D models represent the average fundamental and higher-mode dispersion curves for the chosen source-receiver path as in Yoshizawa and Kennett (2002), Yoshizawa and Ekström (2010), and Visser et al. (2007, 2008).

\subsection{Waveform Modeling}

A synthetic seismogram $(s)$ can be calculated by summation of normal modes $(m)$ of amplitude $A_{m}$ in the frequency domain $(\omega)$ for a 1-D model as follows (e.g. Dahlen and Tromp(1998)):

$$
s(\omega)=\sum_{m} A_{m}(\omega) \exp \left[i \omega \Delta / c_{m}(\omega)\right]
$$

where $c_{m}(\omega)$ is the phase velocity of the mode $m$ at angular frequency $\omega$ and $\Delta$ is the epicentral distance. The relationship between seismograms and their corresponding velocity model is thus highly non-linear. A fully non-linear approach, which does not require any partial derivatives with respect to model parameters or any strong constraints on parameterization, is therefore desirable to perform waveform inversion.

Fortran code Mineos (Masters et al., 2011) can be used to compute synthetic seismograms for a 
reference 1-D model of Earth's interior by normal mode summation. The advantage of such formulation is that it builds a direct connection between seismic waveforms and phase velocities. However, the calculation of normal mode eigenfunctions and eigenfrequencies for a given mantle model is very time-consuming and thus we cannot use the fully non-linear formulation that is Eq. 1 at each iteration of a MCMC scheme. We thus decided to linearize the forward modeling problem in order to overcome the computational speed limitation. As in Yoshizawa and Kennett (2002), we obtained a first synthetic seismogram for a reference model using fully non-linear calculations (Eq. 11). We then used perturbation theory to update the seismogram for other models generated at each iteration of the Markov Chain. For a small perturbation, the change in mode eigenfrequency can be calculated assuming unperturbed eigenfunctions:

$$
\delta \ln (\omega)=\int_{0}^{a}\left(\frac{\delta \alpha}{\alpha}(r) K_{\alpha}(r, \omega)+\frac{\delta \beta}{\beta}(r) K_{\beta}(r, \omega)+\frac{\delta \rho}{\rho}(r) K_{\rho}^{\prime}(r, \omega)\right) d r+\sum_{d} \delta d\left[K_{d}(\omega)\right]_{-}^{+}
$$

where $\delta \ln (\omega)=\delta \omega / \omega, a$ is the radius of the Earth, and $\alpha, \beta, \rho$ and $d$ are P-wave velocity, S-wave velocity, density, and radius of discontinuities, respectively. $K_{\alpha}, K_{\beta}, K_{\rho}^{\prime}$ and $K_{d}$ are the Fréchet derivatives, which relate the change in wave velocities, density, and depth of discontinuity from the reference model to changes in the eigenfrequencies. The Fréchet derivatives can be calculated for each mode using the eigenfunctions determined for the reference model (Woodhouse, 1980).

In theory, all parameters $(\delta \alpha, \delta \beta, \delta \rho$, and $\delta d)$ could be allowed to vary. However, for computational reasons, we decided to only search for shear-wave velocity models. This will unlikely affect the results since P-wave velocity and density have little influence on the phase velocity perturbation in the frequency range considered $(5 \mathrm{mHz}-20 \mathrm{mHz}$ ), as tested by Visser et al. (2008). Similarly, we also neglected perturbations in the depth of mantle discontinuities, which are not well resolved by the data considered (Meier et al., 2009) and including them in the parametrization does not significantly affect the resulting velocity models (Weidner et al., 2017). Correctly accounting for the Moho depth has been shown to be important as it can have strong non-linear effects on waveform modeling and phase velocity calculations (Montagner and Jobert, 1988). We decided to account for deviations from PREM in Moho depth by adopting a reference model that includes crustal constraints from CRUST1.0 (Laske et al., 2013), averaged over the length of the chosen path, and calculating the corresponding shear-wave sensitivity kernels. The partial derivatives employed therefore reflect departures of the Moho from PREM. Hence, Eq. 2 becomes:

$$
\delta \ln (\omega)=\int_{0}^{a} \frac{\delta \beta}{\beta} K_{\beta} d r
$$

The updated frequency can then be converted into phase velocity for a normal mode of angular order 
$l$ using (Jeans, 1923, Dahlen and Tromp, 1998):

$$
c\left(\omega^{*}\right)=\frac{\omega^{*} a}{l+0.5}
$$

where $\omega^{*}$ is the updated eigenfrequency for the mode considered. In Figure 1, we compare synthetic seismograms calculated using the the normal mode summation without linearization (Eq. 1) and the linearized method based on perturbation theory described above (Eq. 3). The results show that the phase velocity information from the linearized perturbation method and the non-linear formulation match well, especially in the period range we are interested in (50-200s).

The rj-MCMC method described below performs a guided Monte Carlo sampling of the model space using the values of the misfit between the real and the synthetic seismograms. The data and the synthetics are compared in different time and frequency windows, which are chosen such that they include the fundamental and several higher modes. We used different time and frequency windows to compare the fundamental and higher mode waveforms. We employed group velocities to choose the beginning and the end of the time windows for the fundamental mode and we employed S- or SS-wave arrival times and group velocities to choose the higher mode time window as in Lebedev and Nolet (2003). For epicentral distance shorter than $35^{\circ}$ the higher mode window start time is set just before the arrival of the $\mathrm{S}$ wave train. For epicentral distance between $35^{\circ}$ and $70^{\circ}$ the start time is just after the S and before the SS wave train. Group velocities can be picked manually by inspection of the waveform or using a frequency-time analysis (Dziewonski et al., 1969). Those windows are summarized in Table 1. An example of time window selection on real data is shown in Fig 2 for an event in Chiapas, Mexico and station HRV in north eastern America.

Because the amplitude of the synthetic seismograms depends on the scalar moments of the event, and because inaccurate scalar moments in catalogues might cause an increase in the data misfit, we implemented an energy equalization method (Lebedev et al., 2005) before calculating the misfit between observed and synthetic seismograms (defined in section 2.2). The aim is to equalize the energy of the synthetic with that of the data by multiplying the amplitude of the synthetics by the following constant:

$$
f^{e q}=\sqrt{\frac{\sum_{t=t_{1}}^{t=t_{2}} d^{2}(t)}{\sum_{t=t_{1}}^{t=t_{2}} s^{2}(t)}}
$$

where $d(t)$ and $s(t)$ are the observed and synthetic seismograms, respectively, filtered in the frequency range considered $(5 \mathrm{mHz}-20 \mathrm{mHz}) . t_{1}$ indicates the beginning of the overtone time window (window 3 defined in Table 1) and $t_{2}$ is the end of the first fundamental mode time window (window 1 in Table 1 . 


\subsection{Bayesian inference}

In a Bayesian formulation, model parameters are described by probability density functions (PDFs), which effectively indicate the uncertainty associated with these parameters. The aim of a Bayesian inference is to quantify the a posteriori probability distribution of all model parameters given some prior information and the data. Bayes' theorem (Bayes, 1763) gives the posterior as:

$$
p\left(\mathbf{m} \mid \mathbf{d}_{o b s}\right) \propto p\left(\mathbf{d}_{o b s} \mid \mathbf{m}\right) p(\mathbf{m})
$$

where $A \mid B$ means $A$ given (or conditional on) $B$, i.e. the probability of having $A$ when $B$ is fixed. $\mathbf{m}$ is the vector of model parameters and $\mathbf{d}_{o b s}$ is the observed data. The term $p\left(\mathbf{d}_{o b s} \mid \mathbf{m}\right)$ is called likelihood function, which shows the probability of observing data $\mathbf{d}_{o b s}$ given a particular model $\mathbf{m}$. The a priori probability of model, $p(\mathbf{m})$, contains what we assume about the model $\mathbf{m}$ before having the observed data. Accordingly, the posterior distribution represents the probability of the model, given the observed data. The parts of the model space that are more frequently required by the data than others manifest with greater posterior probability, and hence are more likely to reflect properties of the Earth (Backus, 1988).

The likelihood function $p\left(\mathbf{d}_{o b s} \mid \mathbf{m}\right)$ describes the probability of data given the current model. In this study, we compared the waveform fit in three different frequency-time windows, and we assumed the data noise in each window follows a multivariate normal distribution with zero mean and covariance matrix $\mathbf{C}_{d_{i}}$, where $i$ is the window index. The likelihood term for each frequency-time window is thus written in this form:

$$
p_{i}\left(\mathbf{d}_{i} \mid \mathbf{m}\right)=\frac{1}{\sqrt{(2 \pi)^{n}\left|\mathbf{C}_{d_{i}}\right|}} \times \exp \left[-\frac{\Phi_{i}(\mathbf{m})}{2}\right]
$$

where $d_{i}$ is the data in window $i, \Phi_{i}(\mathbf{m})$ is the function describing the distance between real data and synthetics predicted by the current model. The general expression for $\Phi_{i}(\mathbf{m})$ considering correlated noise is (Bodin et al., 2012):

$$
\Phi_{i}(\mathbf{m})=\left(G(\mathbf{m})-\mathbf{d}_{i}\right)^{T} \mathbf{C}_{d_{i}}^{-1}\left(G(\mathbf{m})-\mathbf{d}_{i}\right)
$$

where $\mathbf{d}$ represents the measured data vector and $G(\mathbf{m})$ is for the synthetic data predicted by model m.

For simplicity, we assumed Gaussian and uncorrelated noise, in which case the covariance matrix is diagonal. Then the equation above becomes:

$$
\Phi_{i}(\mathbf{m})=M_{i}(\mathbf{m}) / \sigma_{i}^{2}
$$

where $\sigma_{i}$ is the standard deviation of the Gaussian uncorrelated noise in the i-th frequency-time 
window, i.e., the diagonal element of the covariance matrix, and $M_{i}$ is defined by:

$$
M_{i}(\mathbf{m})=\sum_{j=1}^{L_{i}}\left(d_{i}^{j}-f^{e q} s_{i}^{j}\right)^{2}
$$

where $L_{i}$ is the length of the selected time window, $d_{i}$ is the observed data, $s_{i}$ is the synthetics calculated for model $\mathbf{m}$, and $f^{e q}$ is the energy equalization factor defined in Eq. 5. The expression for the likelihood becomes:

$$
p\left(\mathbf{d}_{i} \mid \mathbf{m}\right)=\frac{1}{\left(\sqrt{2 \pi} \sigma_{i}\right)^{n}} \times \exp \left[-\frac{M_{i}(\mathbf{m})}{2 \sigma_{i}^{2}}\right]
$$

The total likelihood function is then the product of the likelihood functions of three frequency-time windows:

$$
p\left(\mathbf{d}_{o b s} \mid \mathbf{m}\right)=p\left(\mathbf{d}_{1} \mid \mathbf{m}\right) p\left(\mathbf{d}_{2} \mid \mathbf{m}\right) p\left(\mathbf{d}_{3} \mid \mathbf{m}\right)
$$

As shown in Eq. 7, the level of noise accounts for theoretical errors, that is, the part of the signal that we are not able to explain with the forward theory and with the chosen parametrization, which is a simplified and discretized representation of reality. Unlike traditional inversion methods in which the noise level is fixed at a presumed level, we use a Hierarchical Baysian approach, treating noise parameters $\sigma_{1}, \sigma_{2}, \sigma_{3}$ as unknowns in the inversion. These noise parameters therefore determine the width of the Gaussian likelihood function and the relative weight given to different frequency-time windows during the inversion. The diagonal element $\sigma_{i}$ is given by a uniform prior distribution, which is explored during the Monte Carlo search. An advantage of this approach is that the weights of the different frequency-time windows are constrained by the data, thereby avoiding an arbitrary choice of noise levels by the user and the risk of overfitting the data. In addition, windows with better waveform fit will naturally have higher weights since the relative weight between different windows is controlled by the data error levels $\sigma_{1}, \sigma_{2}, \sigma_{3}$. We discuss the effects of data noise level in section 3.2

Following Bodin et al. (2012), a uniform prior distribution with relatively wide bounds is adopted here so that the final model will be dominated by the data rather than by prior information. When only independent parameters are considered, the prior probability distribution can be written as the product of three terms:

$$
p(\mathbf{m})=p(\mathbf{c}, \mathbf{v} \mid k) p(k) p(\mathbf{h})
$$

where $\mathbf{c}, \mathbf{v}$ are vectors containing the depth and velocity of each interpolation point, $p(k)$ is the prior on the number of layers, and $p(\mathbf{h})$ is the prior on noise hyper-parameters. Details of the derivation for the expression of $p(\mathbf{m})$ can be found in Bodin et al. (2012). Here, we only show the expression for 
velocity priors at the $i$ th interpolation point $v_{i}$ :

$$
p(\mathbf{v} \mid k)=\left\{\begin{array}{cl}
\frac{1}{\Delta v} & \text { if } V_{\min } \leq v_{i} \leq V_{\max } \\
0 & \text { otherwise }
\end{array}\right.
$$

where $V_{\max }$ and $V_{\min }$ are the upper and lower bounds of velocity, and $\Delta v$ is equal to $\left(V_{\max }-V_{\min }\right)$. Any velocity that falls outside this pre-defined boundaries leads to a null prior. For our study, we chose $\Delta v=10 \%$ of the velocity in the reference model at a given depth.

\subsection{The rj-MCMC method}

The goal of the rj-MCMC method is to generate an ensemble of Earth models distributed according to a target distribution. In our case, we want to find the 1-D shear velocity models that best fit the waveform data to represent the dispersion of multiple modes along a specific source-receiver path.

Most inversion schemes adopt a fixed dimension model space. However, we do not know the complexity of the $V_{S}$ profile, i.e. the dimension of the model space, a priori. One potential drawback of fixing the depth parameterization is that different seismograms may require different number of model parameters to improve the fit and the results could potentially depend on the chosen prior parametrization. Here, we adopted a transdimensional Bayesian inversion, which consists in a sampling-based algorithm that include the number of parameters in the set of unknowns and hence the constraints added on the uncertain parameterization or uncertain data errors can be released. The transdimensional approach lets the data themselves constrain the allowable model complexity instead of having the user choose a model parametrization (e.g., number of layers, or cells) a priori. It is well-known, however, that increasing the complexity of the model parametrization often results in better data fit and introduces parameter trade-offs. One difficulty in inverse problems is to determine which parameters are well resolved and how much complexity is really needed by the data. To avoid this problem, the rj-MCMC sampler, first proposed by Geyer and M $\phi$ ller (1994), is designed to find a parsimonious solution (Malinverno, 2002), i.e. it naturally discourages high dimensional (many layers) models and the least complex explanation or model for an observation is preferred to avoid over-fitting the data.

The rj-MCMC method has been explained in detail in Bodin and Sambridge (2009) and Bodin et al. (2012). Our method is based on theirs except that we used a different depth parameterization. The nodes in the two different parameterizations have, nevertheless, a similar meaning and the derivations are similar to theirs. The main difference comes from the way the velocity models are constructed. In Bodin et al. (2012), each Voronoi nucleus defines its nearest neighbor region as one layer, which has the same velocity as the nucleus within it. By contrast, in our model the shear wave velocity perturbation at a given depth is interpolated from its two nearby nodes. 
In the rj-MCMC method, every new model is generated by iteratively perturbing the last one according to some chosen proposal distribution. There are five types of perturbation based on the parameterization we chose:

(1) Change the velocity of one interpolation point;

(2) Birth: create a new interpolation point;

(3) Death: remove one interpolation point at random;

(4) Move: Randomly pick one interpolation point and move it to a new depth;

(5) Change the noise level;

Here we have employed a slightly different model proposal for Birth than Bodin et al. (2012) due to the different parameterization used here. The other proposals are the same. For the birth step, we added a new interpolation point at depth $c_{k+1}^{\prime}$, and then assigned a new velocity perturbation value $v_{k+1}^{\prime}$ to this new node. This is drawn from a Gaussian proposal probability density:

$$
q\left(v_{k+1}^{\prime} \mid v_{*}\right)=\frac{1}{\theta_{b i r t h \sqrt{2 \pi}}} \exp \left\{-\frac{\left(v_{k+1}^{\prime}-v_{*}\right)^{2}}{2 \theta_{b i r t h}^{2}}\right\}
$$

where $v_{*}$ is the current velocity perturbation value at the depth $c_{k+1}^{\prime}$ where birth takes place. The value of $v_{*}$ can be calculated by a simple interpolation between its two nearest nodes. The standard deviation $\theta_{\text {birth }}$ of the Gaussian distribution is a parameter to be chosen. We refer the reader to Bodin et al. (2012) for the proposal distributions of other types of perturbation.

New models generated from those perturbations are then randomly accepted or rejected according to the acceptance ratio. In order to converge to the target distribution $p\left(m \mid \mathbf{d}_{o b s}\right)$, the acceptance probability from the current model $\mathbf{m}$ to the proposed model $\mathbf{m}^{\prime}, \alpha\left(\mathbf{m}^{\prime} \mid \mathbf{m}\right)$, has to meet the following requirement:

$$
\alpha\left(\mathbf{m}^{\prime} \mid \mathbf{m}\right)=\min \left[1, \frac{p\left(\mathbf{m}^{\prime}\right)}{p(\mathbf{m})} \cdot \frac{p\left(\mathbf{d}_{o b s} \mid \mathbf{m}^{\prime}\right)}{p\left(\mathbf{d}_{o b s} \mid \mathbf{m}\right)} \cdot \frac{q\left(\mathbf{m} \mid \mathbf{m}^{\prime}\right)}{q\left(\mathbf{m}^{\prime} \mid \mathbf{m}\right)} \cdot|\mathbf{J}|\right],
$$

where the matrix $\mathbf{J}$ is the Jacobian of the transformation from $\mathbf{m}$ to $\mathbf{m}^{\prime}$.

The derivations of acceptance probability in rj-MCMC method were given by Bodin et al. (2012), Appendix C. Here we will only describe the ideas briefly and show the equations that differ from theirs. For moves without change of dimension, both the Jacobian term and the ratio of proposal distributions are 1 and the reader can find the same results as Bodin et al. (2012) by inserting Eqs. 11 and 13 into Eq. 16. For the birth and death steps which involve a change of dimension, it can be shown that the Jacobian term is equal to 1 . However, due to the different birth proposal distribution we selected, the expression for the acceptance term for the birth and death steps are different from Bodin et al. (2012). 
For the birth step:

$$
\alpha\left(\mathbf{m}^{\prime} \mid \mathbf{m}\right)=\min \left[1, \frac{\theta_{b i r t h} \sqrt{2 \pi}}{\Delta v} \cdot \exp \left\{\frac{\left(v_{k+1}^{\prime}-v_{*}\right)^{2}}{2 \theta_{b i r t h}^{2}}\right\} \cdot \frac{p\left(\mathbf{d}_{o b s} \mid \mathbf{m}^{\prime}\right)}{p\left(\mathbf{d}_{o b s} \mid \mathbf{m}\right)}\right],
$$

The meaning of $v_{k+1}^{\prime}$ and $v_{*}$ is the same as in 15 . For the death step:

$$
\alpha\left(\mathbf{m}^{\prime} \mid \mathbf{m}\right)=\min \left[1, \frac{\Delta v}{\theta_{b i r t h} \sqrt{2 \pi}} \cdot \exp \left\{-\frac{\left(v_{j}^{\prime}-v_{i}^{\prime}\right)^{2}}{2 \theta_{b i r t h}^{2}}\right\} \cdot \frac{p\left(\mathbf{d}_{o b s} \mid \mathbf{m}^{\prime}\right)}{p\left(\mathbf{d}_{o b s} \mid \mathbf{m}\right)}\right],
$$

where $v_{i}$ is the velocity of the $i$-th interpolation point to be removed at depth $c_{i}$ and $v_{*}$ is the velocity at depth $c_{i}$ in the new structure after the removal of the $i$-th node.

If the newly proposed model is rejected, then the last model is retained for another iteration. The Markov chain is generated via hundreds of thousands of iterations. The first part of the chain (called the burn-in period) is discarded, after which the random walk is assumed to be stationary and starts to sample the model space according to the posterior distribution $p\left(\mathbf{m} \mid \mathbf{d}_{o b s}\right)$. If the algorithm is run long enough, these samples should then provide a good approximation of the structure of Earth as constrained by the data.

This ensemble solution contains many models with variable parameterization, and each $V_{S}$ model in the ensemble corresponds to one dispersion relationship. The expected dispersion is the weighted average through the posterior distribution sampled by the rj-MCMC algorithm. One can use this average to choose one dispersion curve with proper uncertainties for interpretation purpose.

\subsection{Forward modeling and Parametrization}

In this study, we seek a 1-D depth-dependent isotropic shear velocity model to represent the dispersion of multiple modes between a chosen source and receiver. The isotropic nature of the model is assumed mainly for computational reason, but it is a reasonable assumption as demonstrated by Visser (2008) who showed that the differences in the phase velocities calculated assuming isotropy or anisotropy are small. The $V_{S}$ profile is described by a variable number $k$ of interpolation points (Figure 3 ). The vertical position of these points defines the depths at which $V_{S}$ is perturbed and the horizontal position of these points correspond to the amount $V_{S}$ is perturbed relative to a reference model using the velocity prior described by Eq. 14.

We adopted the rj-MCMC algorithm (Bodin and Sambridge, 2009) to explore the model space and sample the distribution of model parameters informed by the data. The procedure of our waveform inversion can be summarized as follows:

(1) Choose path-specific reference 1-D shear velocity model 
12 Haotian Xu and Beghein, $C$.

(2) Calculate normal mode eigenfrequencies for the reference model using MINEOS, and compute a reference synthetic seismogram using normal-mode summation (Eq. 1)

(3) Generate path-specific 1-D shear velocity model by perturbing the reference model according to the proposal distribution of the rj-MCMC scheme;

(4) Compute the synthetic seismogram predicted by the 1-D model using Eq.3,

(5) Calculate the misfit between synthetic and observed waveforms in each window using the $L_{2}$ norm;

(6) Randomly accept or reject the proposed model according to the acceptance ratio;

(7) If the model is accepted, calculate and save the phase velocities predicted by the model using Eq. 4 and generate a new $V_{S}$ model by perturbing the accepted model according to the proposal distribution. If the model is rejected, generate a new $V_{S}$ model by perturbing the previously accepted model according to the proposal distribution;

(8) Repeat steps (4) to (7) to generate the Markov chains;

(9) Gather all the saved phase velocities to determine the distribution of dispersion curves obtained at each period/frequency;

(10) Calculate the mean and standard deviation of these distributions to obtain a measured dispersion curve and uncertainties of phase velocities, respectively;

(11) Estimate the reliability of the dispersion curves (see section 2.5.

\subsection{Reliability Analysis}

In principle, with the proposed method we are able to obtain the phase velocities for any mode at any period since they are calculated from the ensemble of $V_{S}$ models obtained. However, in practice, only some modes at certain periods can be reliably constrained by a single seismogram. It is thus important to estimate the reliability of the measurements in each frequency band for different modes. Here, we adopted a procedure similar to Yoshizawa and Kennett (2002) and Yoshizawa and Ekström (2010) based on the Frequency-Time Analysis method (Dziewonski et al., 1969).

Let us first define the waveform fit $f(\omega, t)$ :

$$
f(\omega, t)=\exp \left[-\frac{S^{\mathrm{mis}}(\omega, t)}{S^{\mathrm{syn}}(\omega, t)}\right]
$$

where $S^{\text {mis }}(\omega, t)$ represents the spectrogram of the difference between real data and the inverted synthetics. $S^{\mathrm{syn}}(\omega, t)$ is the spectrogram of the whole synthetic waveform. Similarly, the relative power of the $j$ th mode $p_{j}(\omega, t)$ is defined as:

$$
p_{j}(\omega, t)=\exp \left[-\frac{S_{j}^{\mathrm{res}}(\omega, t)}{S_{j}^{\mathrm{syn}}(\omega, t)}\right]
$$


where $S_{j}^{\mathrm{syn}}(\omega, t)$ is the spectrogram of the $j$ th mode waveform, and $S_{j}^{\text {res }}(\omega, t)$ is the spectrogram of the residual seimogram calculated as the difference between the full synthetic and the $j$ th mode waveform. In practice, both the frequency $\omega$ and time $t$ are discrete, so the spectrograms can be expressed in matrix form. For example, the element in the $k$ th row and $l$ th column of the spectrogram matrix represents the amplitude of $S\left(\omega_{k}, t_{l}\right)$, where $\omega_{k}$ is the discretized frequency and $t_{l}$ is the center of the $l$ th time window used in the frequency-time domain analysis.

The waveform fit $\mathbf{f}$ measures the difference between the synthetic and real data in the frequencytime domain. It is designed such that $f(\omega, t)=1$ at all frequencies and time when the synthetic seismograms are identical to the observed data, and $\mathbf{f}$ decreases when the waveform fit becomes worse. The relative power $\mathbf{p}_{j}$, which measures the relative power of the $j$ th mode, is designed in a similar way so that $p_{j}(\omega, t)=1$ at all frequencies and time when the $j$ th mode in the seismogram is completely dominant and there is no contribution from other modes, and $\mathbf{p}_{j}$ decreases as the contribution from the $j$ th mode becomes smaller.

For the reliability analysis, we want to consider the overall waveform fit and the relative weight of one single mode at the same time because both factors indicate how well the phase velocities at certain frequencies are constrained by the data. That is, the measurement of the $j$ th mode becomes more reliable if the waveform fit is better, or if the relative weight of the $j$ th mode increases. Following this criteria, we define the reliability parameter as:

$$
r_{j}(\omega)=\sum_{l} p_{j}^{k l} f^{k l}
$$

where $p$ and $f$ are defined in Eqs. 19 and 20, To get the reliability parameter of the $j$ th mode $r_{j}(\omega)$, we first multiply the waveform fit by the relative weight of the $j$ th mode in the F-T domain, and then integrate the resulting matrix with respect to time. In this way, the $r_{j}(\omega)$ is a function of frequency and can be regarded as an indicator of reliability at frequency $\omega$ for the $j$ th mode. Examples of the reliability analysis are shown in section 3 .

\section{TESTS AND RESULTS}

\subsection{Synthetic tests}

We first present a synthetic test to demonstrate that our method can recover an input shear-wave velocity model well (Figures 4 and 5 ). A shear-wave velocity profile generated by perturbing the Preliminary Reference Earth Model (PREM) (Dziewonski and Anderson, 1981) was used to calculate a synthetic seismogram. Figure 4(B) displays the input perturbation $d V_{S}(r) / V_{S}(r)$. It is characterized by $+3 \%$ anomaly relative to PREM at $200 \mathrm{~km}$ depth and $-2 \%$ at $600 \mathrm{~km}$ depth. The synthetic seismogram (Fig- 


\section{Haotian Xu and Beghein, $C$.}

ure 6, representing the velocity of the ground motion, was calculated for a great circle path between the Banda Sea and Beijing, which corresponds to a distance of 5,347km. We used the fundamental mode and the first ten overtones to calculate the synthetics and to invert the synthetic data. The event source parameters were taken from the GCMT catalog (Dziewonski et al., 1981; Ekström et al., 2012) and are shown in Table 2 . Only the vertical component is considered for the waveform fitting. We generated correlated noise by bandpass-filtering a sequence of uncorrelated Gaussian (white) noise, and then added the correlated noise to the synthetic seismogram. This way, the filtered noise sequence is correlated and data points in the sequence are no longer independent The noise level was set at $10 \%$ of the average amplitude of the waveform contained in the third time window as defined in Table 1. which includes higher modes and excludes the fundamental mode.

The inversion of the synthetic waveform data was performed using eight Markov chains starting from different random models, with the length of each chain fixed at 120,000 iterations. To guarantee the convergence of the inversion when sampling the posterior distribution, the first 60,000 iterations in each chain were marked as 'burn-in' and we removed them from the ensemble of sampled models. The ensemble of $d V_{S} / V_{S}$ solutions represented by a color density plot is displayed in Figure 4(A). The brighter color means the algorithm spent more time in that region, and therefore it corresponds to a more likely $V_{S}$ structure at that particular depth. The mean output model calculated from the ensemble of solutions (Figure 4(B)), which can be regarded as the expectation of the "true model", is close to the input model. Figure 5(A) displays the posterior distribution of the number of parameters used by the algorithm during the inversion and demonstrates the parsimony of the transdimensional framework: although we can almost always fit the data better by introducing more parameters, the rj-MCMC method tends to prevent overfitting the data and prefer models of smaller dimensions. In this case the result shows there is a higher probability that the data can be explained by less than eight depth nodes. Figure 5(B) represents the posterior data noise level and shows that fitting the first time window required less data noise than the other two time windows.

A comparison between the input and output seismograms is shown in Figure 6, which shows that the predicted waveform resulting from our inversion fits the input data much better than the waveform predicted by PREM. The misfit between the inverted waveform and the input data is represented in Figure 7 both in the time domain and the F-T domain.

The fundamental mode and first overtone phase velocity dispersion was calculated and compared with the dispersion relationship predicted by the true model (Figure 8). The standard deviation of our measurements at each period were calculated from the ensemble of dispersion curves obtained. We see that the true dispersion curve is within the $95 \%$ likelihood contour of our inversion results at all periods. 
We further applied the reliability analysis described in the previous section to our synthetic test. The relative weight of the first overtone is used as an example to demonstrate the method (Figure 9). The spectrogram of misfit in Figure 7 and the spectrograms of relative weights (like in Figure 9p were multiplied using Eq. 21 and summed along the time domain to obtain the reliability parameters as a function of frequency for each mode (Figure 10 . In order to automate the process, we arbitrarily define the threshold values at 10 for the fundamental mode and 2 for higher modes. For each mode, only periods with reliability parameters larger than the threshold will be kept. As is shown in the synthetic test results, the fundamental mode and the first overtone have high reliability at all periods. The second overtone is also above the threshold at most periods but the third and fourth higher modes only have a short range of reliable results. The fifth overtone cannot be determined reliably in the synthetic test, likely because it was not excited well at those frequencies by the employed seismic source.

Further synthetic tests were performed to verify whether our method is valid for uncorrelated noise (Fig. S1), and for fixed depth nodes, i.e. when the transdimensionality aspect of the software is turned off (Fig. S2). This transdimensionality test showed that for a parameterization with fixed number of parameters, similar to what was implemented by others (Yoshizawa and Kennett, 2002. Yoshizawa and Kennett, 2004; Visser et al., 2007; Visser, 2008; Yoshizawa and Ekström, 2010), we can obtain phase velocity measurements with error bars that include the predictions of the input model, i.e. the "true" data. However, the mean phase velocities deviate from the true values, highlighting the importance of reporting proper uncertainties with the measurements. We also see that the mean phase velocities do not reproduce the input data as well as when the number of parameters is allowed to vary in the inversion. This is visible when comparing Figures S1 and S2. This is also true for the mean velocity model, which is not as close to the input model as the mean model of Figure S1. It should be notes, however, that when the dimension of themodel is fixed, smoothed parameterizations such as B-splines are more commonly employed ((Yoshizawa and Kennett, 2002; Yoshizawa and Kennett, 2004; Visser et al., 2007; Visser, 2008; Yoshizawa and Ekström, 2010)), since they can more easily represent smooth 1-D velocity variations with a limited number of nodes. In addition, we note that the computational time when the transdimensionality is turned on was not very different from when it was turned off.

We additionally tested the effect of the data noise level by underestimating it (Fig. S3) and overestimating it (Fig. S4) compared to the input data. These figures demonstrate that the prior data noise level can have large influence on the inverted phase velocities as well as their estimated uncertainties: when the noise level is underestimated, the posterior uncertainties on the estimated phase velocities become smaller but the input data are not well represented by those uncertainties as they lie outside (or 
close to) two standard deviations. When the noise level is overestimated, however, the uncertainties on the phase velocities are much larger but the mean is close to the input data. We therefore argue that overestimating the noise level leads to more reliable measurements, albeit with larger uncertainties, and reduces the possibility of overfitting the data.

\subsection{Application to real data}

In this section we present the phase velocity inversion results for two paths across western and eastern Australia. Figure 11 shows the locations of the events and stations. Both selected events have depths larger than $50 \mathrm{~km}$. The source parameters of the two events are listed in Table 2 . The epicentral distance for the western path is $4,036 \mathrm{~km}$, and for the eastern path is $4,012 \mathrm{~km}$. Although a non-linear inversion method is used here to calculate phase velocities from the $S$-wave velocity models, it can still be preferable to use a reference model that is as close as possible to the true structure of the region studied. This is mainly because our method to calculate the synthetic seismograms is not fully non-linear for computational reasons (see section 2). The forward modeling part of the algorithm was linearized, and we search for model parameters $d V_{S} / V_{S}$ between $-5 \%$ and $5 \%$ around a reference model in order to reduce errors caused by the linearization. Here, we adopted the 3-D shear-velocity model of Debayle et al. (2016). We averaged the $V_{S}$ profiles extracted from the reference model along each station-event pair, and used this average as a reference model for the specific 1-D path considered. The prior data noise level was a uniform distribution between 1 and $500 \mathrm{~nm} / \mathrm{s}$ for each time window.

The posterior distribution of $d V_{S} / V_{S}$ models for the western Australia path is shown in Figure 12, followed by the number of parameters and data noise distributions (Figure 13, the waveform fit (Figure 14), the reliability analysis (Figure 15), and the estimated phase velocity dispersion curves (Figure 16). The inverted $d V_{S} / V_{S}$ structure (Figure 12 displays a modest positive deviation from the reference model between $50 \mathrm{~km}$ and $200 \mathrm{~km}$ depth as well as below $400 \mathrm{~km}$ depth. The uppermost positive anomaly is consistent with a thick lithosphere as expected in a cratonic area. The number of velocity parameters required by the data is around four, which demonstrates the parsimony of the algorithm, and the posterior data noise distributions have relatively narrow Gaussian distributions with a standard deviation much lower than the assumed prior noise level. Overall, the fundamental mode in the first window $(5-10 \mathrm{mHz})$ is the lowest, followed by the higher modes window.

The synthetic waveform calculated from the mean velocity model fits the real data well (Figure 14), and we see that phase velocities for the fundamental mode has reliability parameters larger than the predefined threshold, 10, at all periods considered (Figure 15). For the first overtone, the reliable measurements are in the period range $92-200 s$, with the threshold set at 2 . Similarly, the second overtone phase velocities were estimated to be reliable in the period range $58-148 s$. The reliability 
parameters of the third overtones is between $60-88 s$. Finally, a small portion $(50-57 s)$ of the fourth overtone was found to be reliable.

For the eastern Australia path, we repeated the procedure described above. The results are shown in Figs. 17, 21. We see a strong positive $V_{S}$ anomaly compared to the reference model at about $60 \mathrm{~km}$ depth and a negative $V_{S}$ anomaly at $120-220 \mathrm{~km}$ depth. Our solution can be interpreted as a thin lithosphere (about 100km thick) with slightly positive velocity anomalies and a low velocity layer that may coincide with the asthenosphere (Fig. 22(B)), consistent with Phanerozoic geological features and with previous studies (Yoshizawa and Kennett, 2015). It should also be noted, however, that our $V_{S}$ solutions are not directly comparable with 3-D models, but rather can be regarded as a 1-D approximation of the real Earth structure along the selected path. Figure 18 shows that the number of parameters required to fit the data is between four and eight and the data noise level is clearly the lowest for the fundamental mode filtered between $5 \mathrm{mHz}$ and $10 \mathrm{mHz}$ (window 1). Overall the noise level is much higher for the eastern Australia path than for the western Australia path. With Figure 20. we show that the fundamental mode and the first four overtones can be reliably retrieved: the fundamental mode has high reliability at 50-150s. The first and second overtones have large reliability at longer periods $(60-200 s, 54-200 s)$. The reliable periods for third and fourth overtones are $50-83 s$ and $50-61 s$, respectively. Measurements for the modes and periods estimated reliable are shown in Fig. 21. This figure also displays phase velocities measured using S40RTS (Ritsema et al., 2011) as a reference instead of the Debayle et al. (2016) model to test the dependence of our method upon the reference model. The results show no significant dependence on the reference model. The reliability tests were not strongly affected by the reference model either (not shown here).

The mean absolute $V_{S}$ model were also plotted for parths and compared to PREM (Figure 22(A)). This figure shows a clear difference between the eastern and western paths in the top $200 \mathrm{~km}$, beyond which the two velocity profiles become more similar.

In this study we used the centroid moment tensor from the GCMT catalog. We acknowledge, however, that for some paths dispersion measurements performed using waveform modeling at single stations can be affected by uncertainties in source parameters. While quality control can be applied to filter out unreliable earthquake sources by comparing source parameters from different earthquakes in the same region or by comparing different source catalogues, joint inversions of structure and source parameters are preferable (Valentine and Trampert, 2012). A future implementation of our technique will include source parameter among the unknowns in order account for possible errors in the source parameters. 


\section{CONCLUSION}

We applied the rj-MCMC technique to the non-linear problem of measuring fundamental and higher mode Rayleigh wave phase velocity dispersion using waveform modeling. The use of higher mode surface waves in depth inversions of seismic velocities and anisotropy is very valuable as it increases the vertical resolution of tomographic models in the upper mantle and transition zone. The forward problem consisted in calculating synthetic seismograms by normal mode summation using computer program Mineos and a linearized approximation to the calculation of normal mode eigenfrequencies. The rj-MCMC method enabled us to find a distribution of 1-D shear velocity models that best fit the waveform and represent the dispersion of multiple modes along a specific source-receiver path. An advantage of this technique is that the algorithm can decide how much data noise is needed to fit the data without overfitting them. The posterior noise distributions can then be used as an indicator of the quality of the waveform fit within each frequency-time window. The data noise was thus treated as an unknown and different noise levels were applied to the different time windows considered. The resulting distribution of velocity models was used to calculate a posterior distribution of dispersion curves, from which a mean and standard deviation were obtained for different modes. A reliability analysis was then performed following Yoshizawa and Kennett (2002) to assess which higher modes were reliably separated.

With synthetic tests, we showed that the newly developed method presented here can extract the first four overtones and the fundamental mode at most perios between 50 and 200s, but that the third and fourth overtones can only be measured reliably at relatively short periods and in a narrower period band (50-100s for $n=3$ and 50-60s for $n=4)$. Measurements performed for real data along two paths sampling western and eastern Australia showed similar results: the fundamental mode and first two overtones were measured reliably over most frequencies considered, and the third and fourth overtones could be extracted and their dispersion measured over a narrower frequency band and for shorter periods. We also demonstrated that the measured phase velocities do not strongly depend on the reference model used to calculate the initial seismogram.

While computationally intensive, the technique presented here has several advantages. It allows the user to sample the model space without requiring any explicit regularization other than specifying the bounds of the model space explored. In addition, because it is transdimensional and parsimonious, it can include data noise and the number of parameters among the unknowns without overfitting the data. It therefore lets the data themselves control the complexity of the solution. The reliability of mode separation can be assessed and quantitative uncertainties on the dispersion curves of each mode can be readily obtained. This provides useful information on the data to seismologists who use these measurements to model three-dimensional seismic velocity and anisotropy. Our technique can also 
be easily adapted to include source parameters among the unknowns and perform joint inversions of model structure and source parameters to account for the effect of source uncertainties on the measured phase velocities. A future implementation of the method will also include perturbations in Moho depth as it can have an important effect on waveform modeling for paths that travel mostly across continental areas. We also note that the phase velocities are not the only products of this technique. One could also envision using the resulting path-averaged velocity models and uncertainties and combine them to obtain a three-dimensional velocity model.

\section{ACKNOWLEDGMENTS}

We wish to thank two anonymous reviewers for their helpful comments as well as Thomas Bodin for sharing his original MCMC code. The code was modified by H.X. to incorporate program MINEOS, which is freely available on the CIG website at http://www.geodynamics.org/. Calculations were made on C.B.'s computer cluster, which was funded by NSF grant \#0949255. Figures were made using Matlab and the Python plotting library Matplotlib (Hunter, 2007).

\section{REFERENCES}

Backus, G. E. (1988), Bayesian inference in geomagnetism, Geophysical Journal, 92 125-142. doi:10.1111/j.1365-246X.1988.tb01127.x

Bayes, T. (1763), An essay towards solving a problem in the doctrine of chances, Philos. Trans. R. Soc. London, 53, 370-418. [Reprinted in Biometrika, 45, 295-315, 1958.]

Beucler, E., Stutzmann, E. and Montagner, J. P. (2003), Surface wave higher-mode phase velocity measurements using a roller-coaster-type algorithm, Geophys. J Int., 155(1), 289-307.

Bodin, T., and M. Sambridge (2009), Seismic tomography with the reversible jump algorithm, Geophys. J Int., 178(3), 1411-1436.

Bodin, T., M. Sambridge, H. Tkalčić, P. Arroucau, K. Gallagher, and N. Rawlinson (2012), Transdimensional inversion of receiver functions and surface wave dispersion, J. geophys. Res., 117, B02301, doi:10.1029/2011JB008560.

Cara, M. (1973), Filtering of dispersed wavetrains, Geophys J Roy Astron Soc., 33(1), 65-80.

Cara, M. (1978), Regional variations of higher Rayleigh-mode phase velocities: A spatial-filtering method, Geophys. J Int., 54(2), 439-460.

Cara, M. (1979), Lateral variations of S velocity in the upper mantle from higher Rayleigh modes, Geophys. J Int., 57(3), 649-670.

Cara, M. and Lévêque, J. J. (1987), Waveform inversion using secondary observables, Geophys. Res. Lett., 14(10), 1046-1049. 
Crampin, S. (1964), Higher Modes of Seismic Surface Waves: Preliminary Observations, Geophys. J Int., 9(1), 37-57, doi:10.1111/j.1365-246X.1964.tb06313.x

Dahlen, F. A. and Tromp, J. (1998), Theoretical global seismology, Princeton University Press, Princeton.

Debayle, E. (1999), SV-wave azimuthal anisotropy in the Australian upper mantle: preliminary results from automated Rayleigh waveform inversion, Geophys. J Int., 137, 747-754, doi:10.1046/j.1365246x.1999.00832.x

Debayle, E., Dubuffet, F., and Durand, S. (2016), An automatically updated S-wave model of the upper mantle and the depth extent of azimuthal anisotropy, Geophys. Res. Lett., 43, 674-682, doi:10.1002/2015GL067329.

Dziewonski, A., Bloch, A., and Landisman, M. (1964), A Technique for the Analysis of Transient Seismic Signals, Bull. seism. Soc. Am., 59(1), 427-444.

Dziewonski, A. and Anderson, D.M. (1981), Preliminary reference Earth model, Phys. Earth Planet. Inter., 25(4), 297-356.

Dziewonski, A. M., T.-A. Chou and J. H. Woodhouse (1981), Determination of earthquake source parameters from waveform data for studies of global and regional seismicity, J. geophys. Res., 86, 2825-2852. doi:10.1029/JB086iB04p02825

Ekström, G., M. Nettles, and A. M. Dziewonski (2012), The global CMT project 2004-2010: Centroid-moment tensors for 13,017 earthquakes, Phys. Earth Planet. Inter, 200-201, 1-9, doi:10.1016/j.pepi.2012.04.002

Geyer, C., and J. M $\phi$ ller (1994), Simulation procedures and likelihood inference for spatial point processes, Scand. J. Stat., 21(4), 359-373.

Hunter, J. D. (2007), Matplotlib: A 2D graphics environment, Computing in science \& engineering, 9(3), 90-95.

Jeans, J. H. (1923), The Propagation of Earthquake Waves, Proc. R. Soc. Lond. Series A, Containing Papers of a Mathematical and Physical Character, 102(718), 554-574, doi:10.1098/rspa.1923.0015

Laske, G. and Widmer-Schnidrig, R. (2015), Normal Mode \& Surface Wave Observations, in Treatise on Geophysics: Seismology and Structure of the Earth, Vol. 1 (2nd edition), 117-165.

Laske, G., G. Masters, Z. Ma, and M. Pasyanos (2013), Update on CRUST1.0 - A 1-degree Global Model of Earth's Crust, in EGU General Assembly Conference Abstracts, EGU General Assembly Conference Abstracts, vol. 15, p. 2658.

Lebedev, S. and Nolet, G. (2003), Upper mantle beneath Southeast Asia from S velocity tomography, J. geophys. Res., 108(B1), doi:10.1029/2000JB000073.

Lebedev, S., G. Nolet, T. Meier, and R. D. van der Hilst (2005), Automated multimode inversion of surface and S waveforms, Geophys. J Int., 162, 951964.

Lerner-Lam, A. L. and Jordan, T. H. (1983), Earth structure from fundamental and higher-mode waveform analysis, Geophys. J Int., 75(3), 759-797.

Lévêque, J. J., Cara, M. and Rouland, D. (1991), Waveform inversion of surface wave data: test of a new tool for systematic investigation of upper mantle structures, Geophys. J Int., 104(3), 565-581.

Li, X. and Romanowicz, B. (1995), Comparison of global waveform inversions with and without considering 
cross-branch modal coupling, Geophys. J Int., 121, 695-709.

Li, X. and Romanowicz, B., (1996), Global mantle shear velocity model developed using nonlinear asymptotic coupling theory, J. geophys. Res., 101,22 245-22 273.

Maggie, A., Debayle, E., Priestley, K., and Barruol, G. (2006), Azimuthal anisotropy of the Pacific region, Earth Planet. Sci. Lett., 250, 53-71, doi:10.1016/j.eps1.2006.07.010

Malinverno, A. (2002), Parsimonious Bayesian Markov chain Monte Carlo inversion in a nonlinear geophysical problem, Geophys. J Int., 151(3), 675-688

Masters, G., Woodhouse, J.H., Freeman, G. (2011), Mineos v1.0.2 [software], Computational Infrastructure for Geodynamics, https://geodynamics.org/cig/software/mineos/

Meier, U., Trampert, J., and Curtis, A. (2009), Global variations of temperature and water content in the mantle transition zone from higher mode surface waves, Earth Planet. Sci. Lett., 282(1-4), 91-101, doi:10.1016/j.eps1.2009.03.004

Montagner, J.-P. (1986), Regional There-dimensional structures using long-period surface waves, Annales Geophysicae, 4(B3), 283-294

Montagner, J.-P., and Jobert, N. (1986), Vectorial tomography?ii. Application to the Indian Ocean, Geophys. J. R. astr. Soc., , 94(2), 309-344, doi:10.1111/j.1365-246X.1988.tb05904.X

Nolet, G. (1975), Higher Rayleigh modes in western Europe, Geophys. Res. Lett., 2(2), 60-62, doi:10.1029/GL002i002p00060

Nolet, G. (1990), Partitioned waveform inversion and two-dimensional structure under the network of autonomously recording seismographs, J. geophys. Res., 95(B6), 8499-8512, doi:10.1029/JB095iB06p08499

Ritsema, J., Deuss, A., van Heijst, H.J., and Woodhouse, J.H. (2011), S40RTS: a degree-40 shear-velocity model for the mantle from new Rayleigh wave dispersion, teleseismic traveltime and normal-mode splitting function measurements, Geophys. J Int., 184(3), 1223-1236, doi:10.1111/j.1365-246X.2010.04884.X

Roult, G. and Romanowicz, B. (1984), Very long-period data from the GEOSCOPE network: Preliminary results on great circle averages of fundamental and higher Rayleigh and Love modes, Bull. seism. Soc. Am., 74(6), 2221-2243

Sambridge, M. (1999a), Geophysical inversion with a Neighbourhood Algorithm -I. Searching a parameter space, Geophys. J Int., 138, 479-494.

Sambridge, M. (1999b), Geophysical inversion with a Neighbourhood Algorithm -II. Appraising the ensemble, Geophys. J Int., 138, 727-746.

Stutzmann, E. and Montagner, J. P. (1993), An inverse technique for retrieving higher mode phase velocity and mantle structure, Geophys. J Int., 113, 669-683.

Montagner, J. P., Romanowicz, B. and Karczewski, J. F. (1994), A first step toward an oceanic geophysical observatory, Eos, Transactions American Geophysical Union, 75(13), 150-154.

Valentine, A.P. and Trampert, J. (2012), Assessing the uncertainties on seismic source parameters: Towards realistic error estimates for centroid-moment-tensor determinations, Phys. Earth Planet. Inter., 210-211, 36-49, doi:10.1016/j.pepi.2012.08.003. 
van Heijst, H.J. and Woodhouse, J.H. (1997), Measuring surface-wave overtone phase velocities using a modebranch stripping technique, Geophys. J Int., 131, 209-220.

van Heijst, H.J. and Woodhouse, J.H. (1999), Global high-resolution phase velocity distributions of overtone and fundamental-mode surface waves determined by mode branch stripping,, Geophys. J Int., 137, 601-620.

Visser, K., Lebedev, S., Trampert, J. and Kennett, B.L.N (2007), Global Love wave overtone measurements, Geophys. Res. Lett., 34, L03302, doi:10.1029/2006GL028671.

Visser, K., (2008), Monte Carlo search techniques applied to the measurements of higher mode phase velocities and anisotropic surface wave tomography, $P h D$ thesis, Utrecht University, the Netherlands.

Weidner, E.C., Xu, H., Beghein, C., Huang, Q., and Schmerr, N. (1980), Toward a new 3-D radial anisotropy model of the upper mantle and transition zone, Fall meeting, AGU, New Orleans, Louisiana, 11-15 Dec 2017

Woodhouse, J.H. (1980), The coupling and attenuation of nearly resonant multipiers in the Earth's free oscillation spectrum, Geophys. J. R. Astr. Soc., 61, 261-283.

Yoshizawa,K. and Kennett, B.L.N. (2002), Non-linear waveform inversion for surface waves with a neighbourhood algorithm: application to multimode dispersion measurements, Geophys. J Int., 149, 118-133.

Yoshizawa,K. and Kennett, B.L.N. (2004), Multi-mode surface wave tomography for the Australian region using a three-stage approach incorporating finite frequency effects, J. geophys. Res., 109, B02310, doi:10.1029/2002JB002254.

Yoshizawa,K. and Ekström, B.L.N. (2010), Automated multimode phase speed measurements for highresolution regional-scale tomography: application to North America, Geophys. J Int., 183(3), 1538-1558, doi:10.1111/j.1365-246X.2010.04814.x.

Yoshizawa,K. and Kennett, B.L.N. (2015), The lithosphere-asthenosphere transition and radial anisotropy beneath the Australian continent, Geophys. Res. Lett., 42, 3839-3846, doi:10.1002/2015GL063845. 
Table 1. Selection of frequency-time windows. The first and second window indices correspond to the fundamental mode and the third window is for higher modes. The start and end times for the fundamental mode are determined by the group velocities $U$ indicated in the table and the event epicentral distance $\Delta$. For the higher modes, the beginning of the time window is determined by the S- or SS-wave arrival times. The end of the window is determined by $\Delta / U$.

\begin{tabular}{cccc}
\hline Window index & Frequency(mHz) & Start & End \\
\hline 1 & $5-10$ & $U=4.45 \mathrm{~km} / \mathrm{s}$ & $U=2.95 \mathrm{~km} / \mathrm{s}$ \\
2 & $10-20$ & $U=4.30 \mathrm{~km} / \mathrm{s}$ & $U=3.20 \mathrm{~km} / \mathrm{s}$ \\
3 & $10-20$ & $S$ or $S S$ & $U=4.30 \mathrm{~km} / \mathrm{s}$ \\
\hline
\end{tabular}

Table 2. Source parameters from GCMT Catalog. Event 200503021042A was used for the synthetic test. 200910151211A was for the western Australia path and 201004172315A for the eastern Australia path.

\begin{tabular}{cccccccc}
\hline ID & date & time & $\operatorname{lat}\left({ }^{\circ}\right)$ & $\operatorname{lon}\left({ }^{\circ}\right)$ & $\operatorname{depths}(\mathbf{k m})$ & $M_{0}$ (dyne-cm) & strike,dip,rake \\
\hline 200503021042A & $2005 / 03 / 02$ & $10: 42: 16.9$ & -6.54 & 129.99 & 196.1 & $5.73 \mathrm{e}+26$ & $41,88,55$ \\
$200910151211 \mathrm{~A}$ & $2009 / 10 / 15$ & $12: 11: 19.8$ & -3.04 & 139.45 & 105.1 & $1.34 \mathrm{e}+25$ & $296,83,145$ \\
$201004172315 \mathrm{~A}$ & $2010 / 04 / 17$ & $23: 15: 27.9$ & -6.82 & 147.30 & 62.9 & $2.19 \mathrm{e}+25$ & $268,50,91$ \\
\hline
\end{tabular}



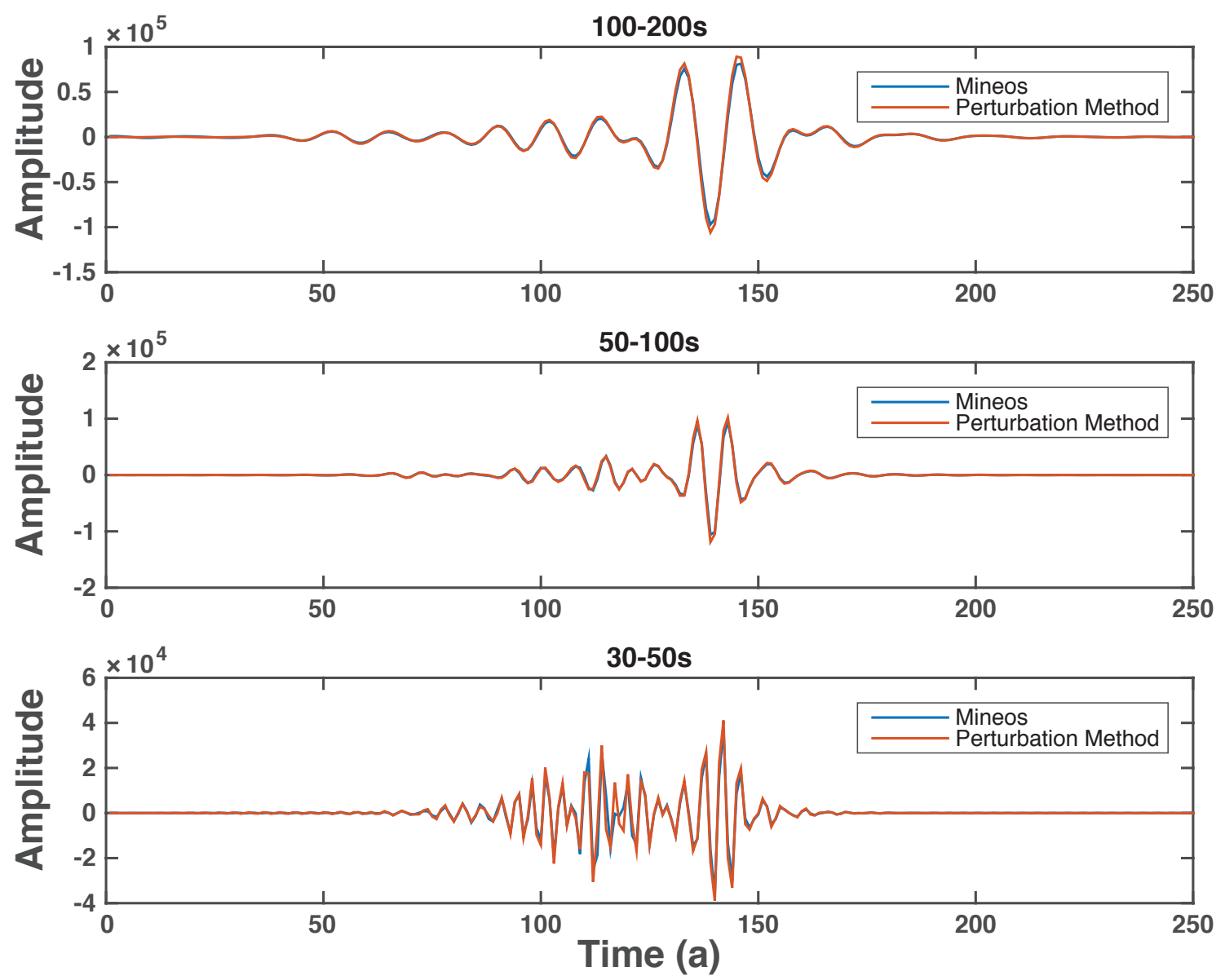

Figure 1. Comparison between synthetic seismograms calculated with normal mode summation using Mineos without linearization (blue) and with our linearized method (red) in three different period ranges: 100-200 s (top), 50-100 s (middle), and 30-50 s (bottom). The shear-velocity model used to compute the seismograms is shown in Figure 3. 

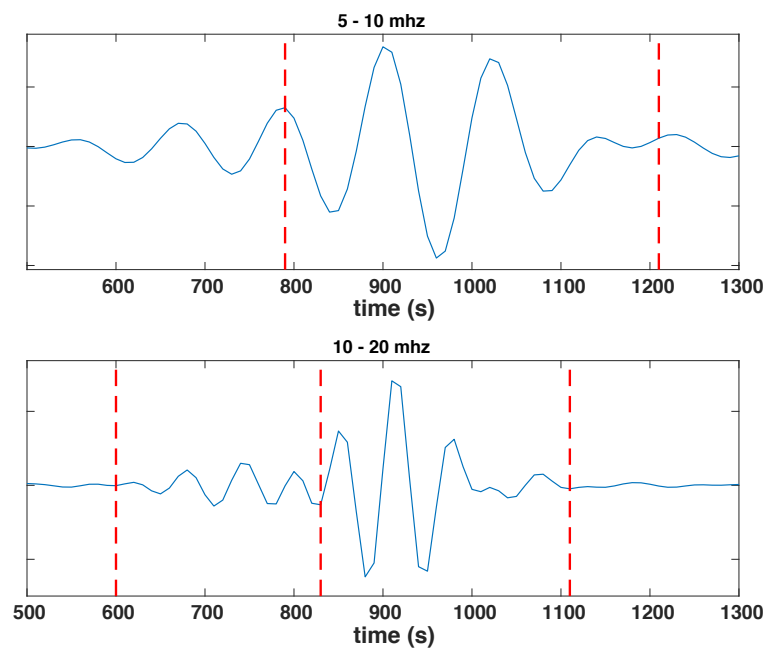

Figure 2. An example of our automatic time window selection for a path across North America. The waveform is for the observed data. The station, HRV, is located in northeastern America. The event was in Chiapas, Mexico and occurred on 6 July, 2007. The time window in the upper panel is window 1 as defined in Table 1 . The time window on the right side of the lower panel is for the fundamental mode (window 2) and the time window on the left of the lower panel is the higher modes window (window 3).

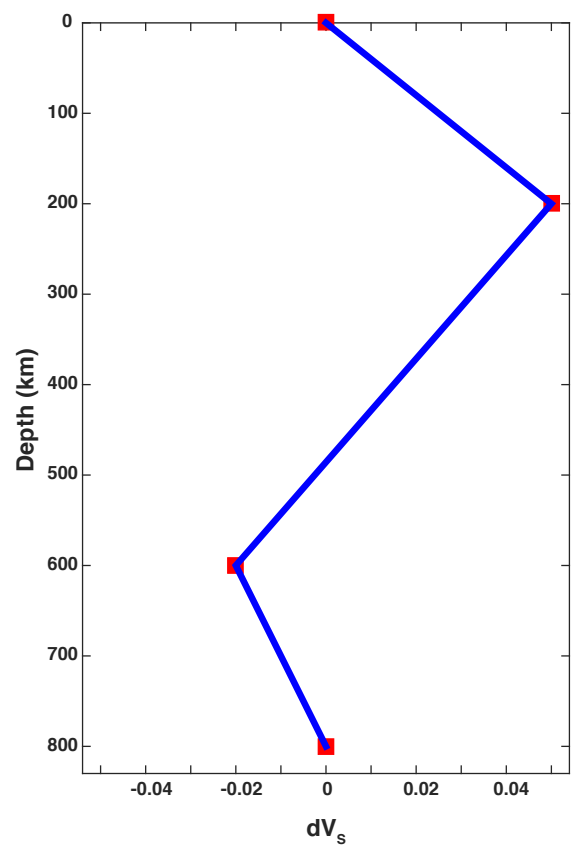

Figure 3. Example of model parametrization. The red squares denote the points at which the velocity model is perturbed. The complete $d V_{S}$ profile is obtained by linear interpolation between those points. 

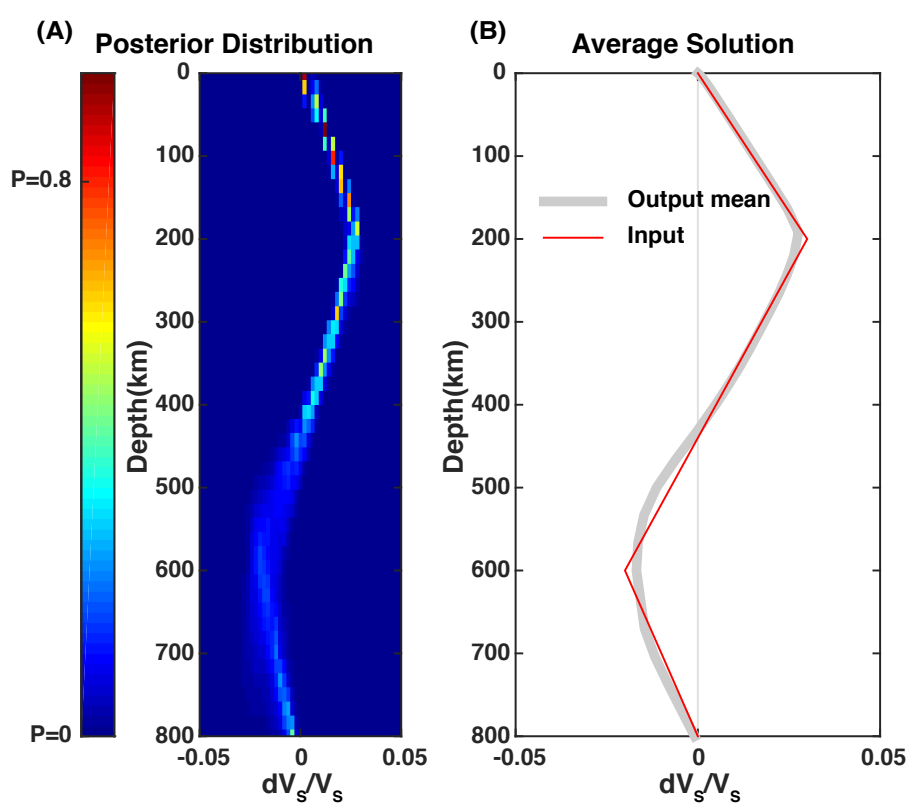

Figure 4. (A) Color density plot representing the ensemble of solutions obtained by inversion of the synthetic seismogram that was calculated with the input model shown in (B); The posterior mean model resulting from this distribution of solutions is also displayed in (B)

(A)

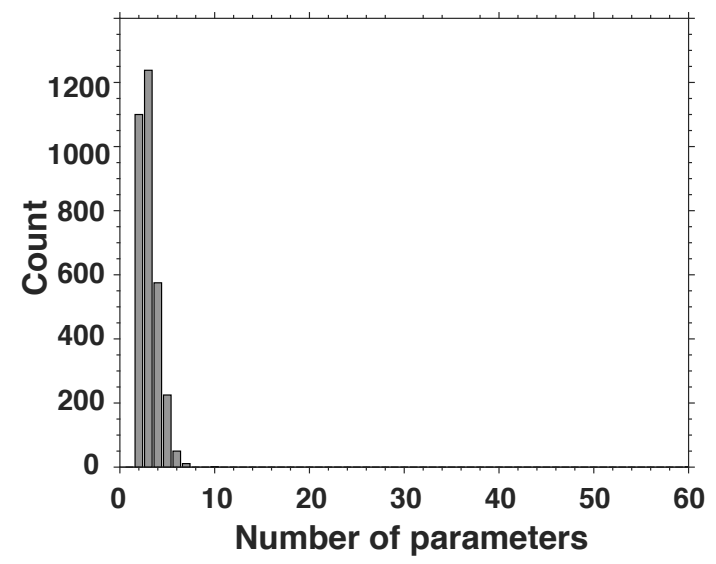

(B)
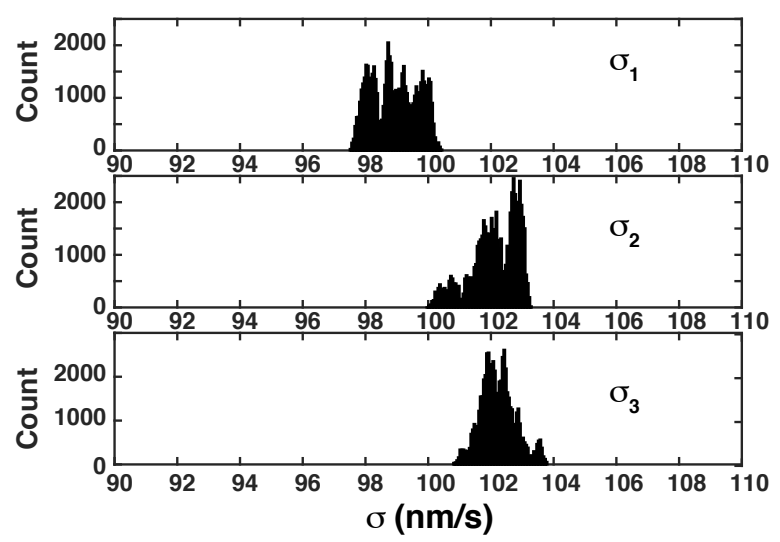

Figure 5. (A) Posterior distribution for the number of parameters in the synthetic test of Figure 4, (B) Posterior distribution for the data noise level for each of the three time windows considered. 

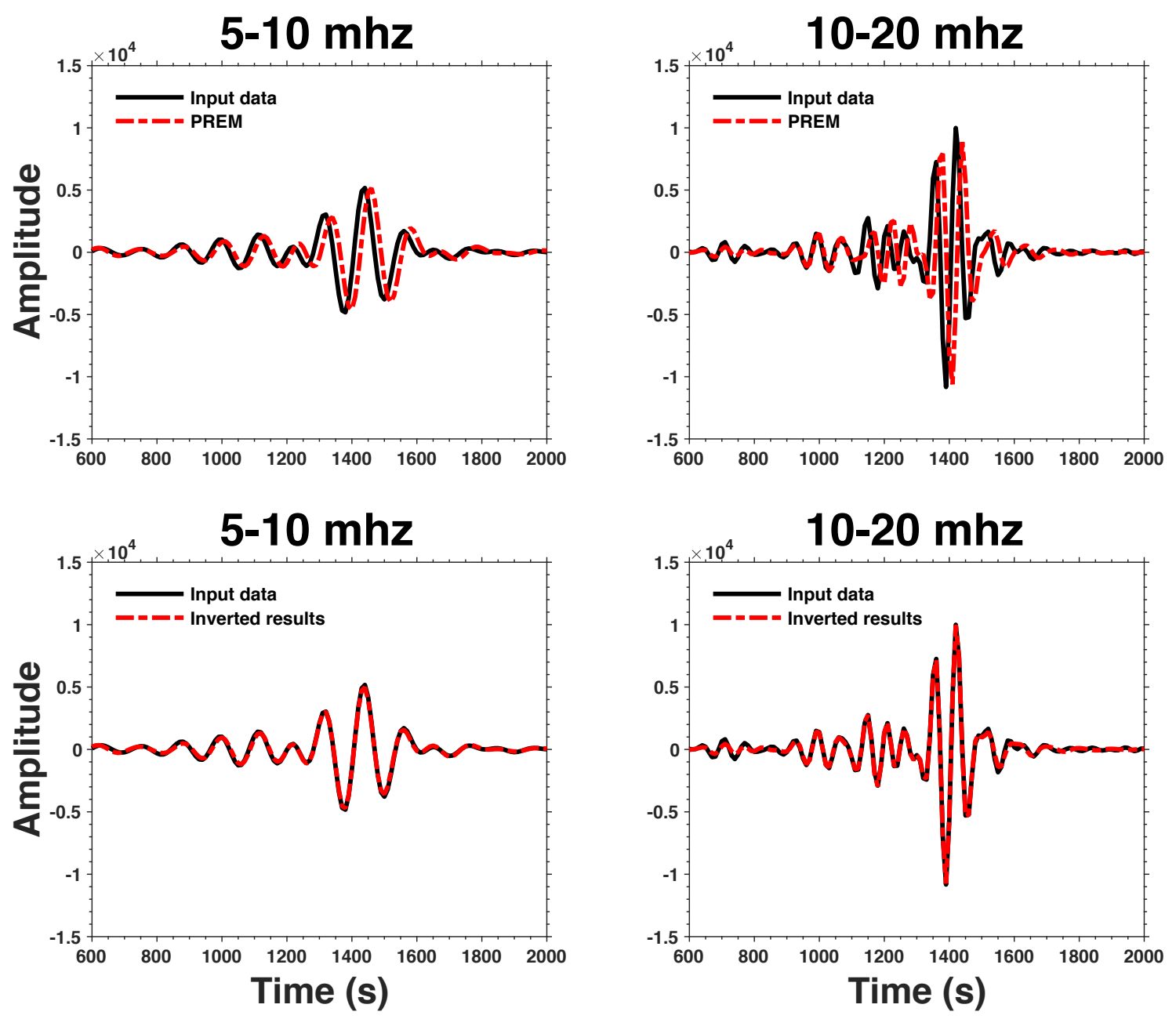

Figure 6. Comparison between input synthetic waveform and the waveform predicted by PREM (top) and by the mean model resulting from our inversion (bottom). The seismograms shown here represent the velocity of the ground motion. The input seismogram was calculated for the true model shown in Figure 4 . Two frequency bands, as indicated in table 1, are considered separately. The waveforms match is much improved after inversion. 


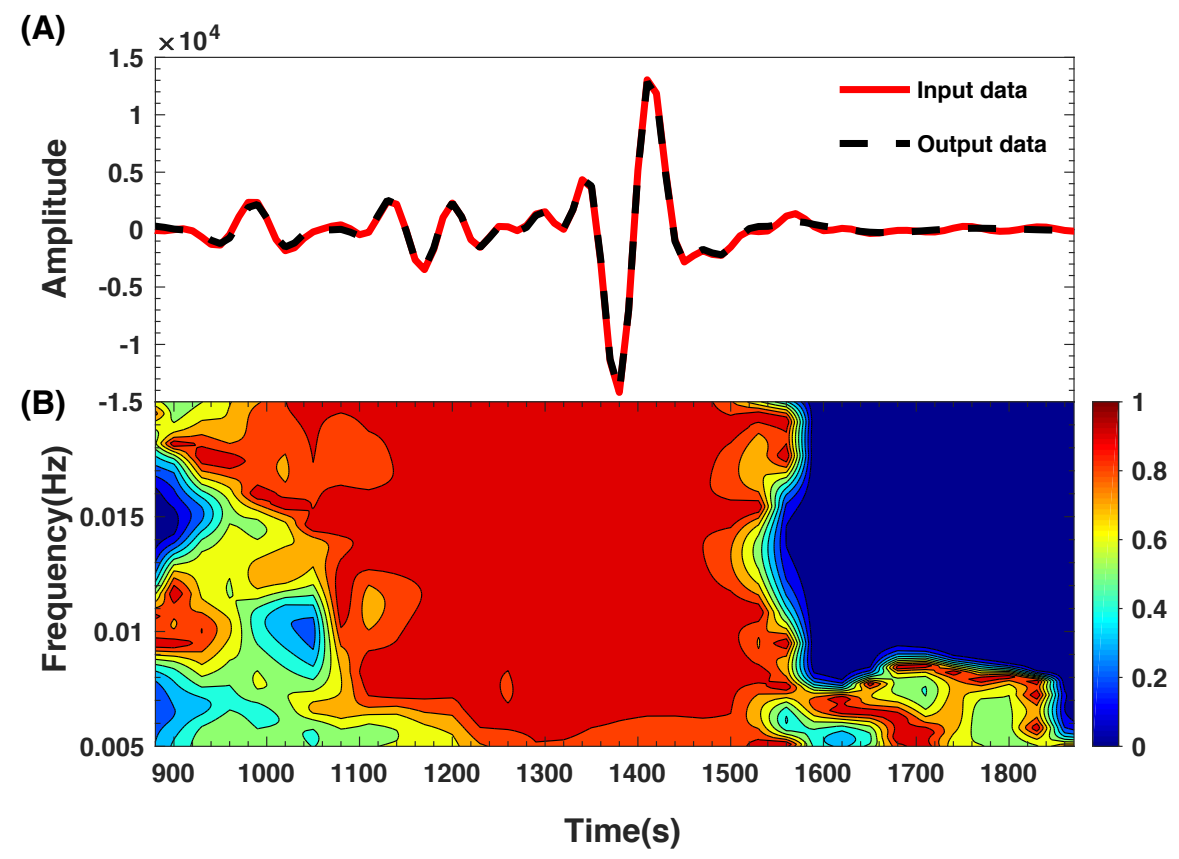

Figure 7. (A) Comparison between the output and input synthetic data in the time domain filtered in the 50s200s period range; (B): Spectrogram of the waveform fit $\mathrm{f}(\omega, t)$, as defined in equation 19 The regions with warmer colors correspond to better waveform fit in the F-T domain. 


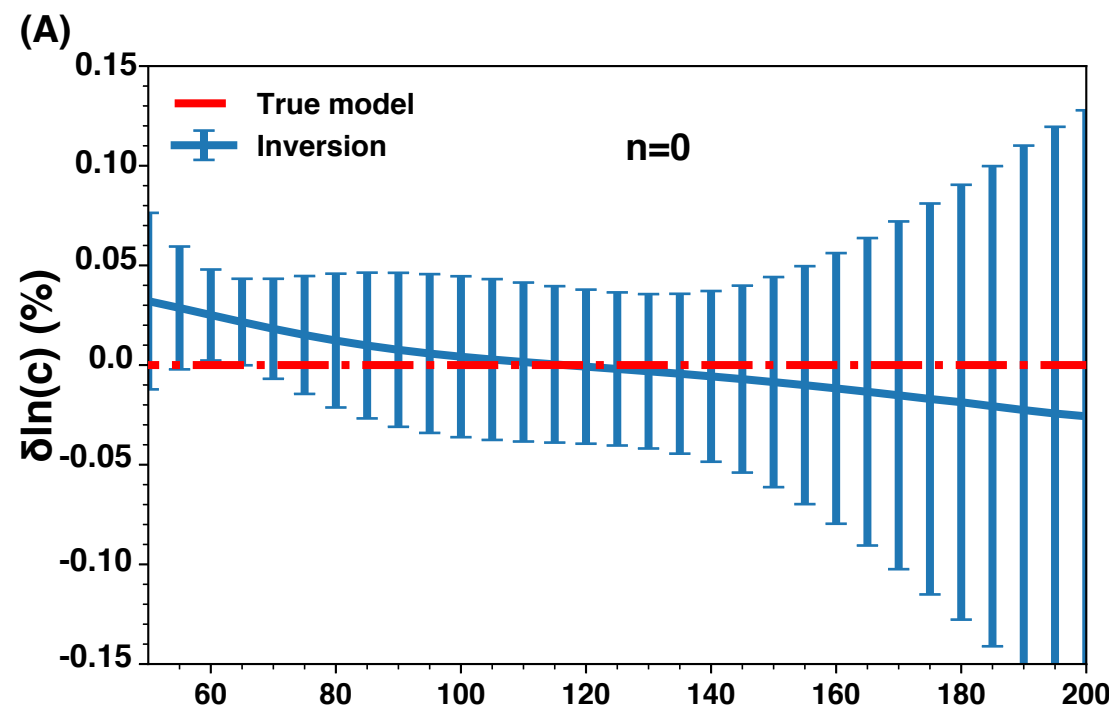

(B)

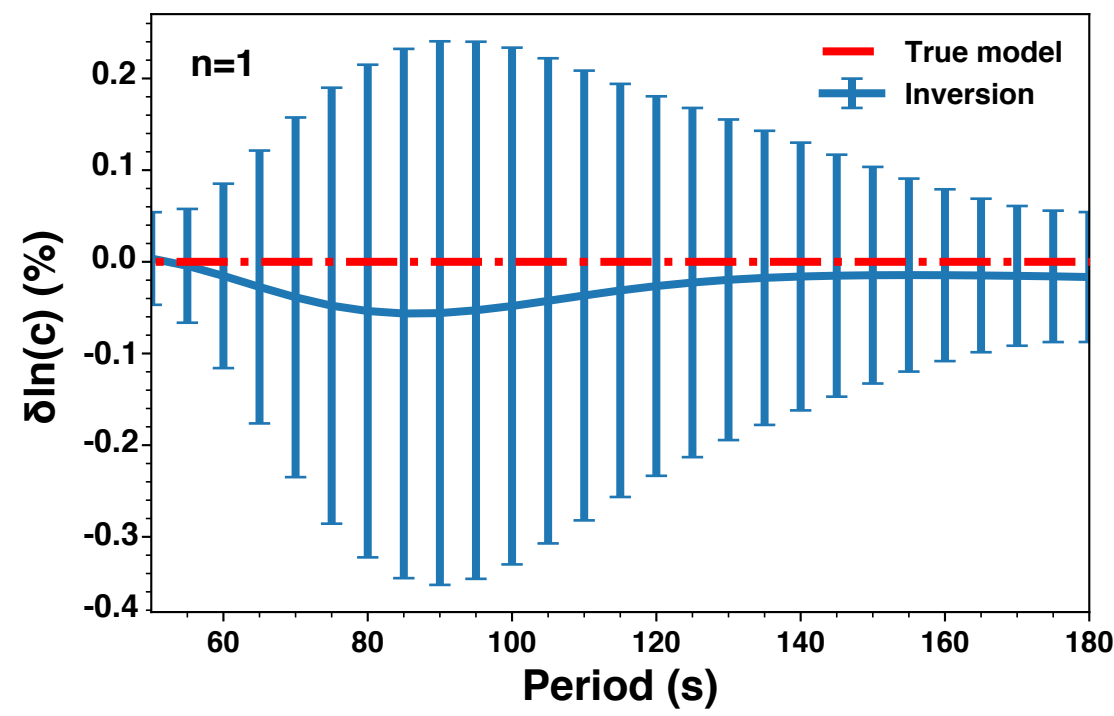

Figure 8. Inverted fundamental mode (A) and first overtone (B) phase velocity dispersion and uncertainties (blue) compared to the dispersion curve calculated for the true model (red). The uncertainties shown here correspond to $2 \sigma$ obtained from the posterior distribution. 


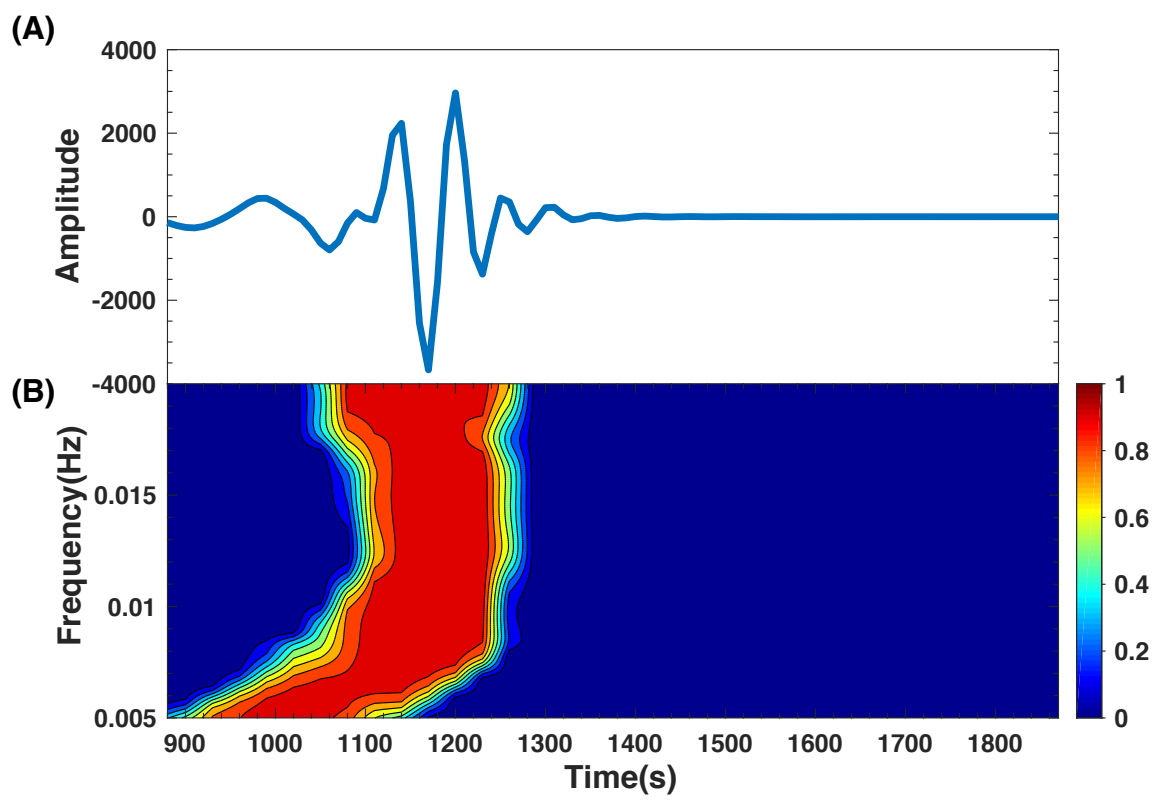

Figure 9. (A) Synthetic waveform of the first overtone calculated from the inverted 1-D $V s$ profile and filtered in the 50s-200s period range; (B): Spectrogram $\mathrm{P}_{1}(\omega, t)$ of the relative weight of the first overtone in the synthetic test, as defined in equation 20 . The regions with warmer colors in the figure represent larger weights in the F-T domain.
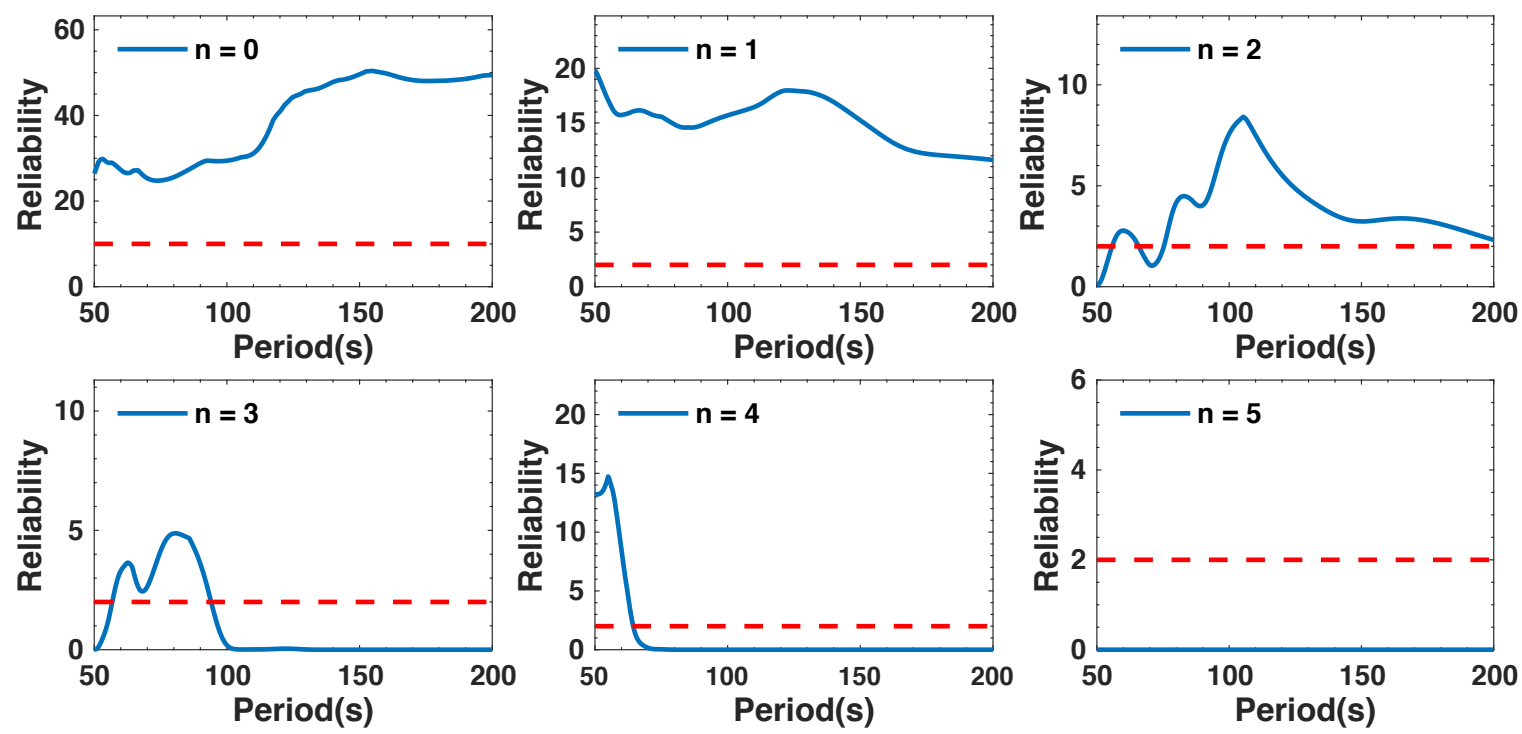

Figure 10. Reliability parameters as a function of frequency for the fundamental mode $(n=0)$ and the first five overtones in the synthetic test. The thresholds we defined for each mode are denoted by the red dashed lines. Only periods at which the reliability parameters are above the threshold are kept as reliable inversion results. 


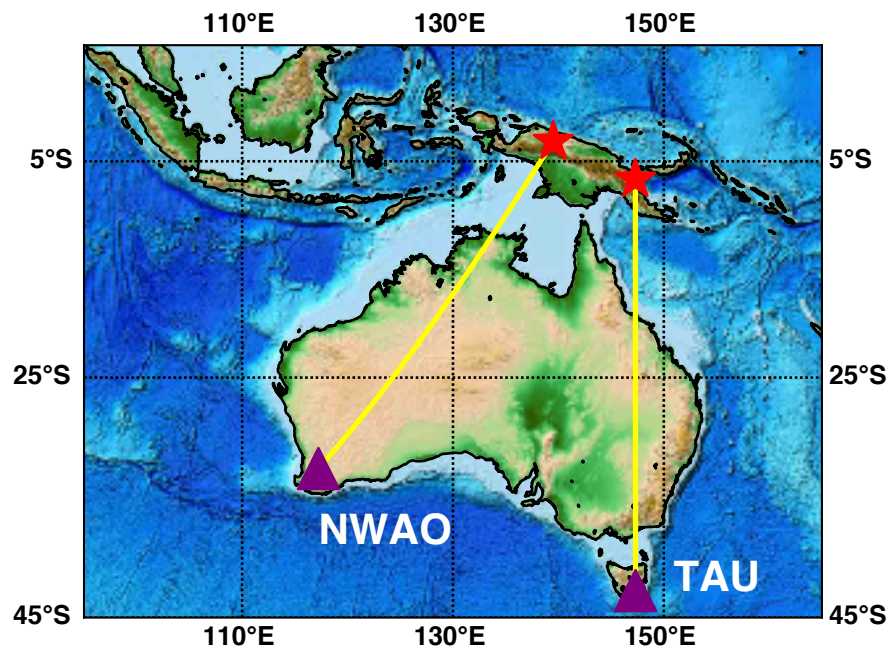

Figure 11. Locations of events (stars) and stations (triangles) used for the real data test. The yellow lines show the great circle paths from sources to receivers for western and eastern Australia.
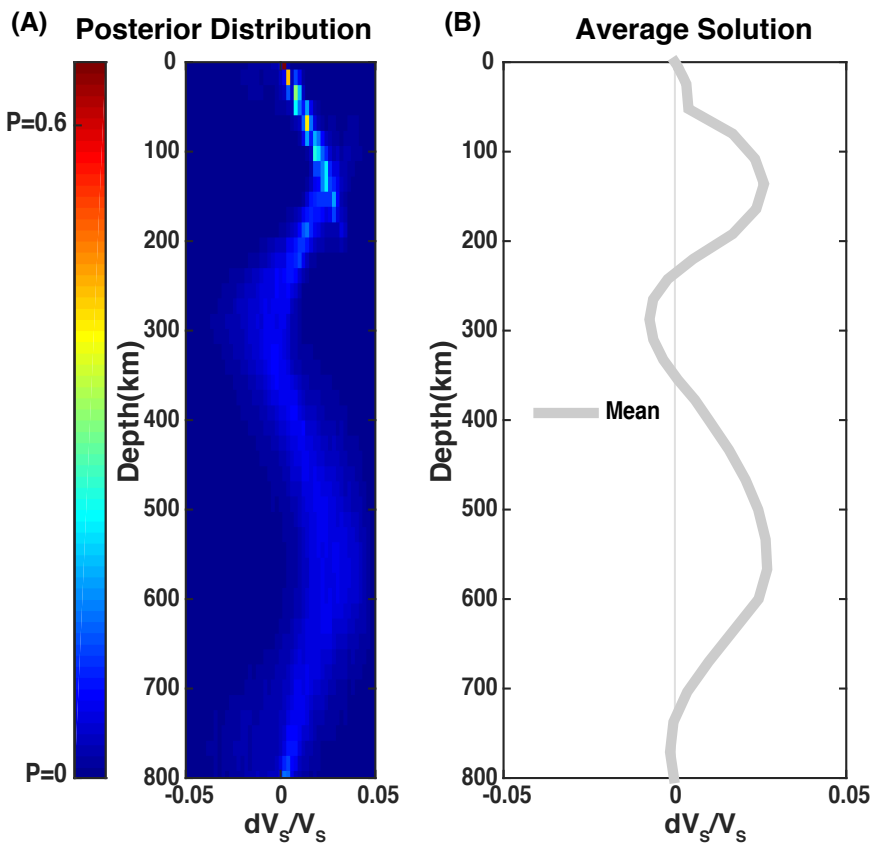

Figure 12. (A): Posterior distribution of relative velocity perturbations with respect to Debayle et al. (2016) for the western Australia path; (B) Resulting mean $d V_{S} / V_{S}$ model 

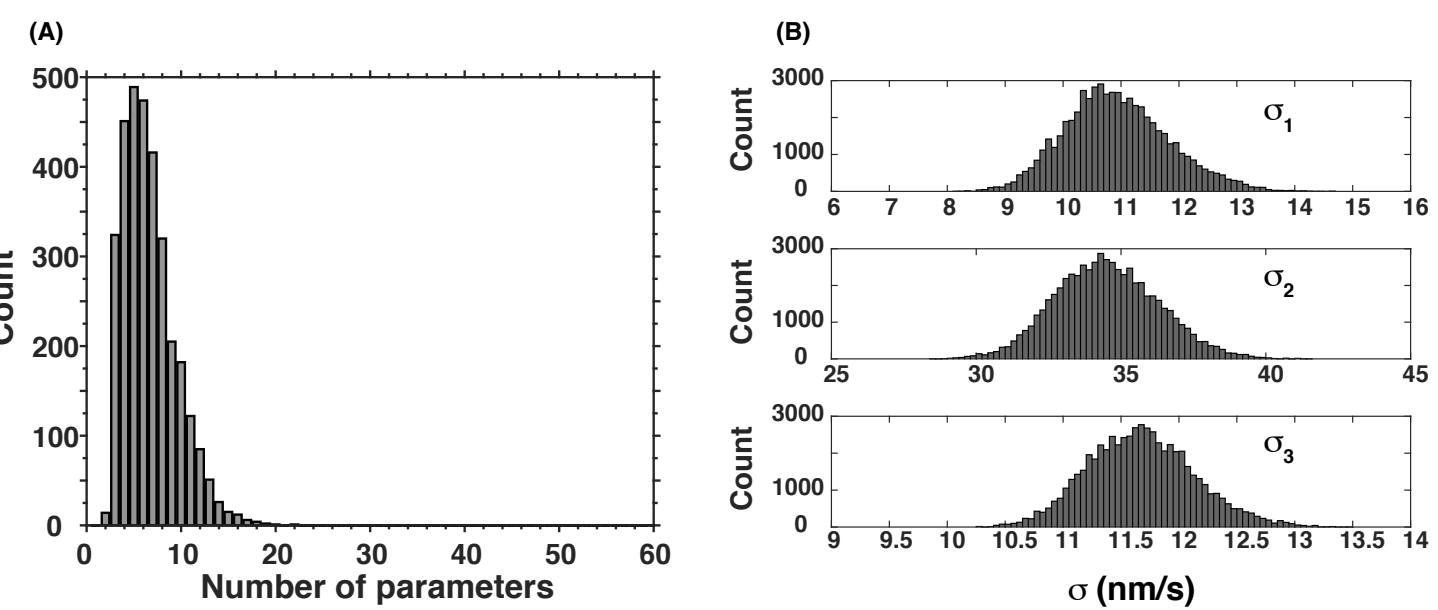

Figure 13. (A) Posterior distribution of the number of velocity parameters for the western Australia path; (B) Posterior data error distribution for each of the three time windows. Note the different scales on the horizontal axes.

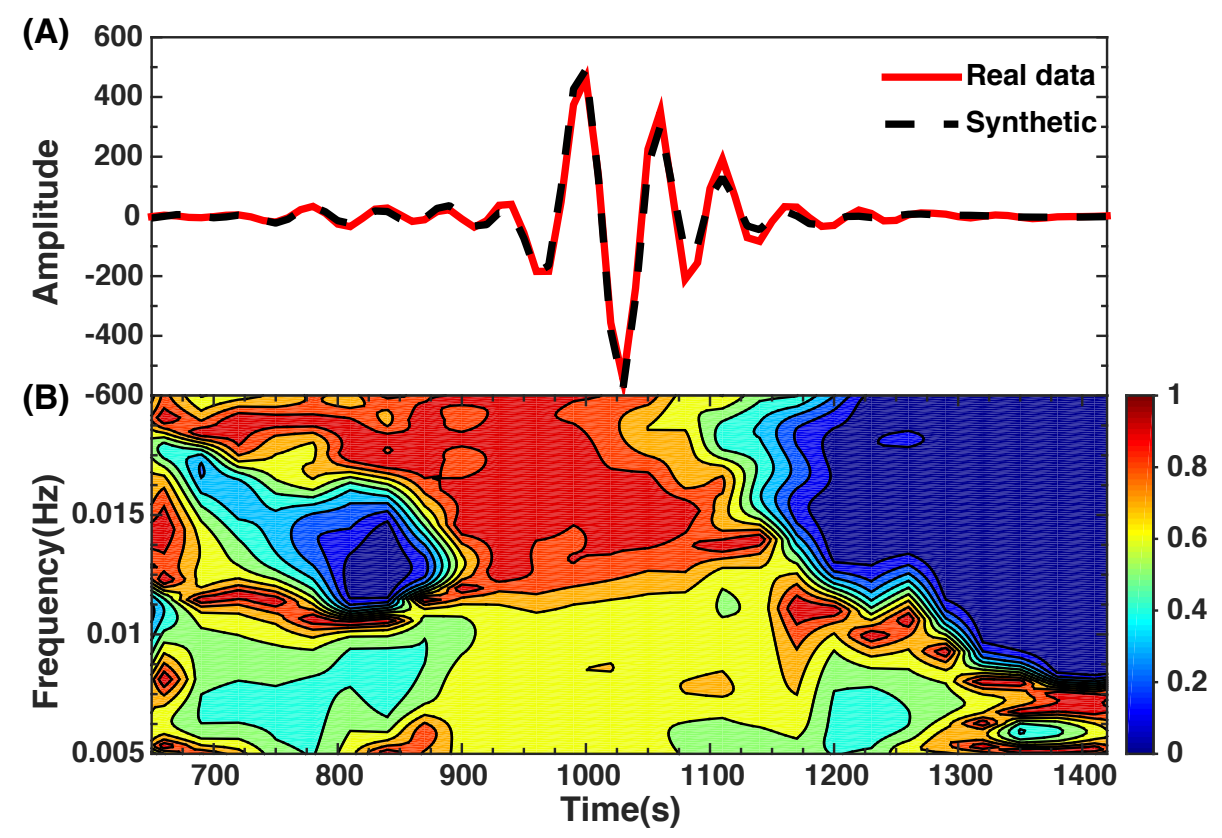

Figure 14. The same as Figure 7 but for the western Australia path. (A): real data and synthetic seismogram filtered in the 50s-200s period range and calculated using the inverted mean model of Figure 12, (B) F-T analysis of the misfit as defined in equation 19 . 

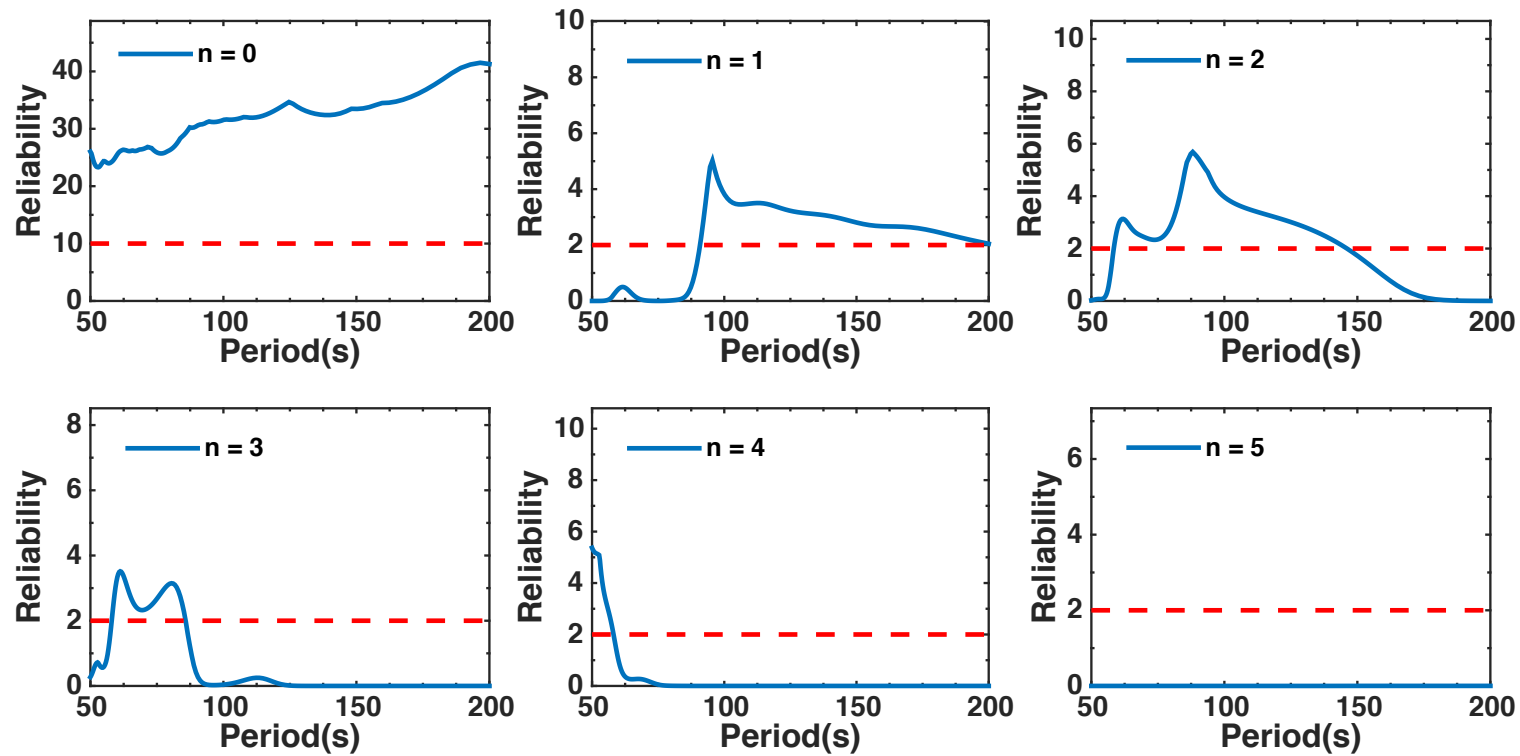

Figure 15. Reliability parameters for the western Australia path. See Figure 10 for details. 

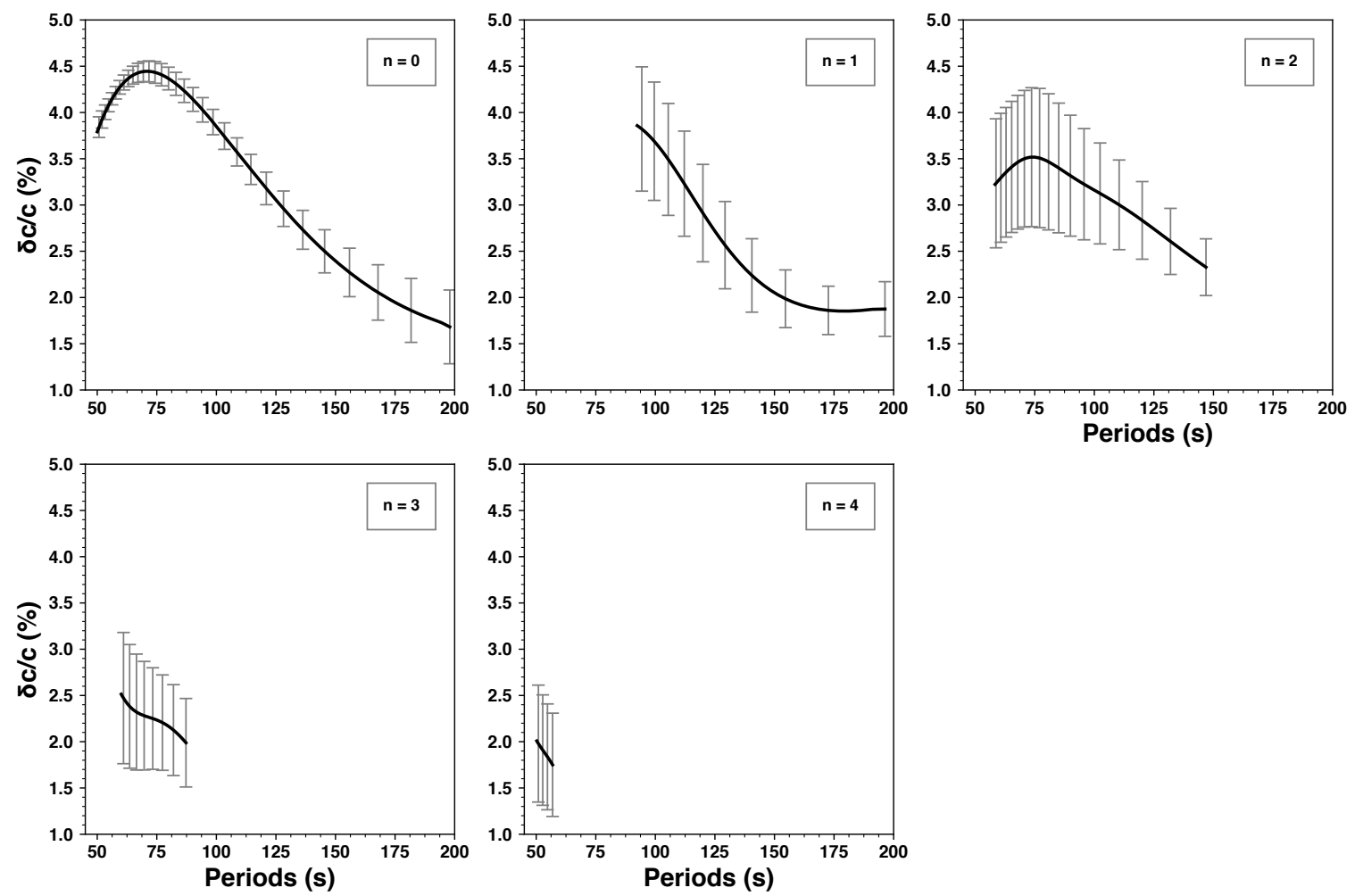

Figure 16. Measured phase velocities anomalies for the western Australia path and the modes and periods that were estimated to be reliable (Fig. 15). The measurements were converted from perturbations with respect to the average version of the 3-D reference model into perturbations with respect to PREM. Uncertainties correspond to $2 \sigma$. 

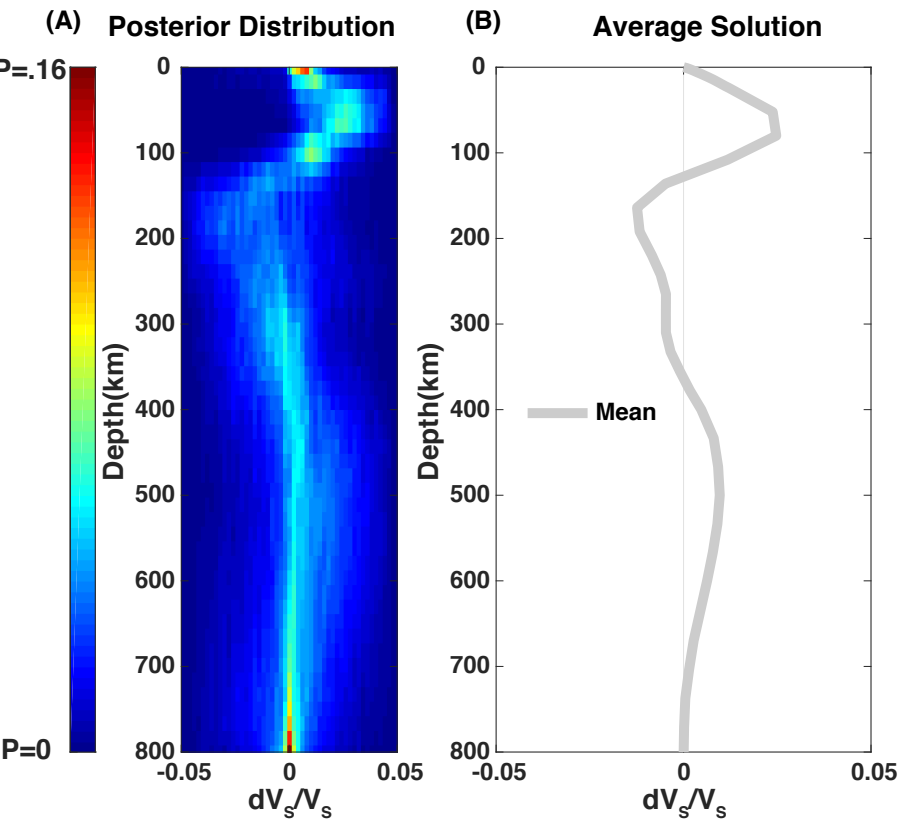

Figure 17. Posterior ensemble of solutions for the eastern Australia path. See Fig. 4 for details.

(A)

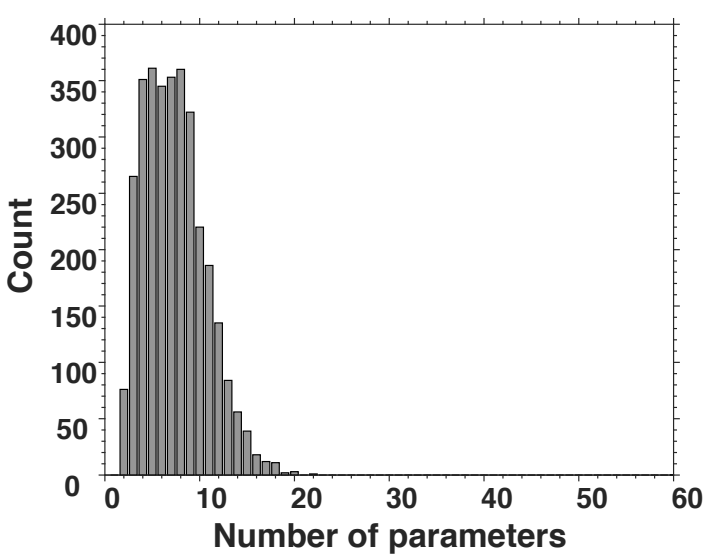

(B)
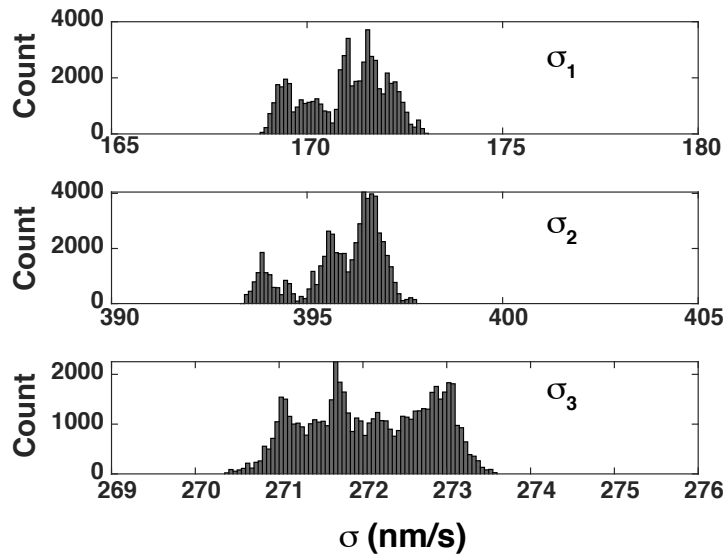

Figure 18. (A) Posterior ensemble for the number of parameters for the eastern Australia path; (B) Posterior noise distribution for each the the three time windows. Note the different horizontal scales. 


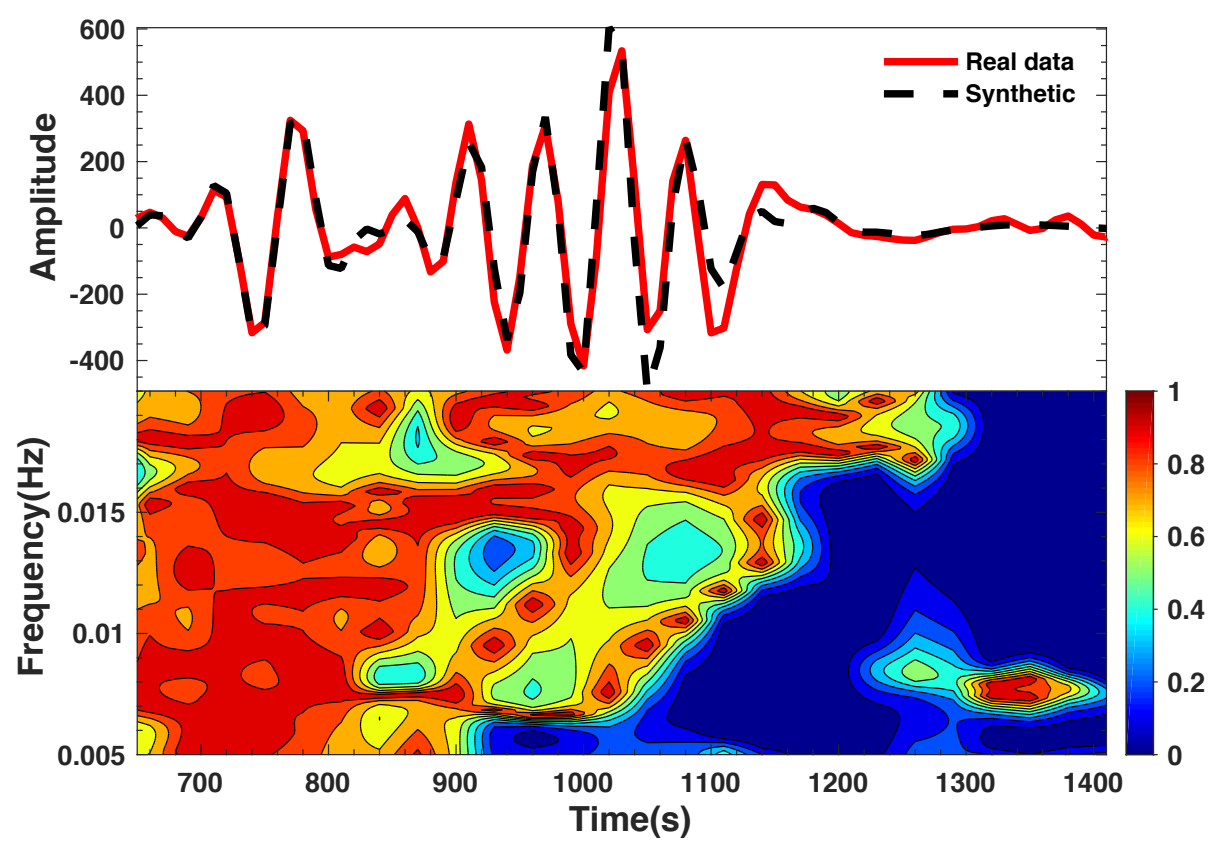

Figure 19. Real data and synthetic seismogram filtered in the 50s-200s period range and calculated using the inverted mean model of Figure 17 (top); F-T analysis of the misfit as defined in equation 19 (bottom).
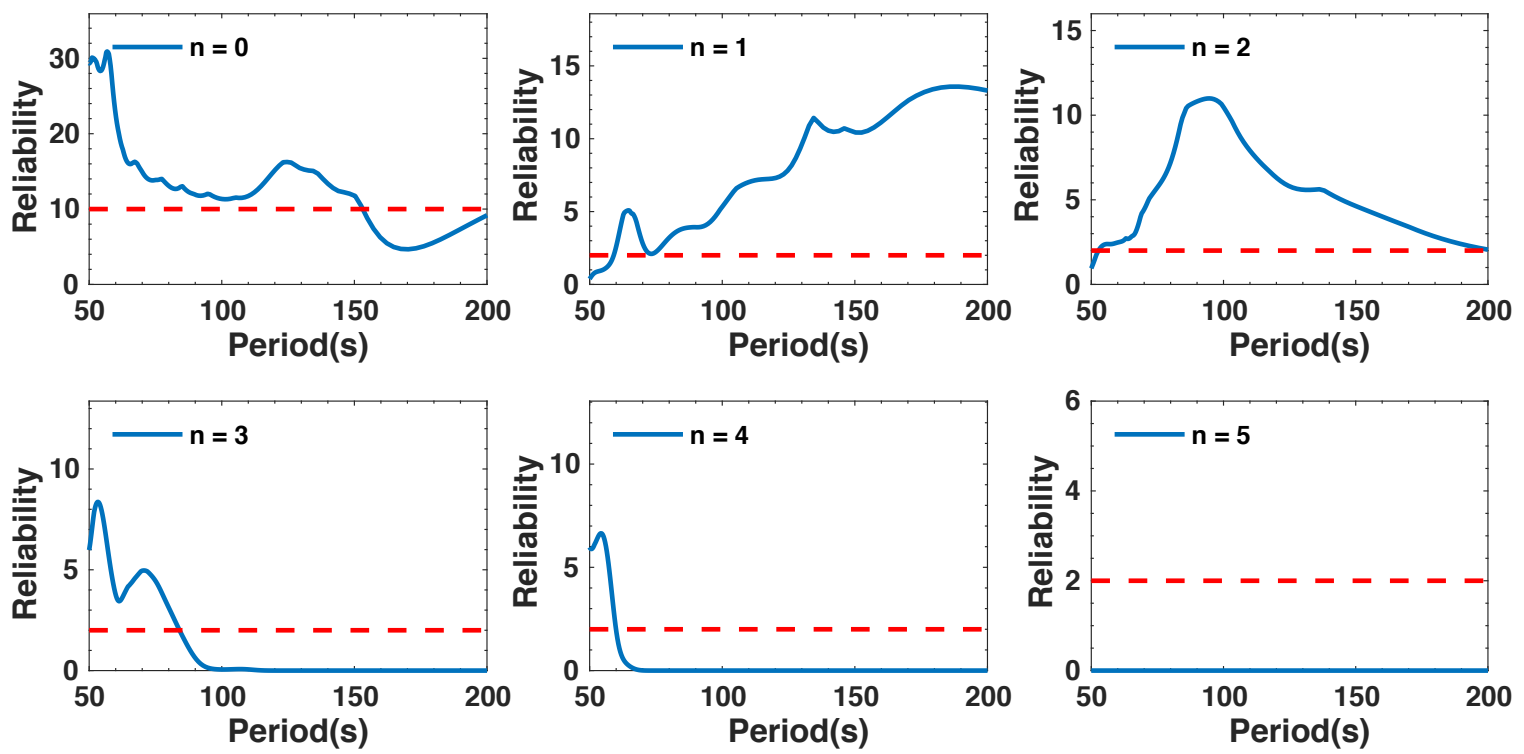

Figure 20. Reliabiliy test for the eastern Australia path. See Fig. 10 for details 

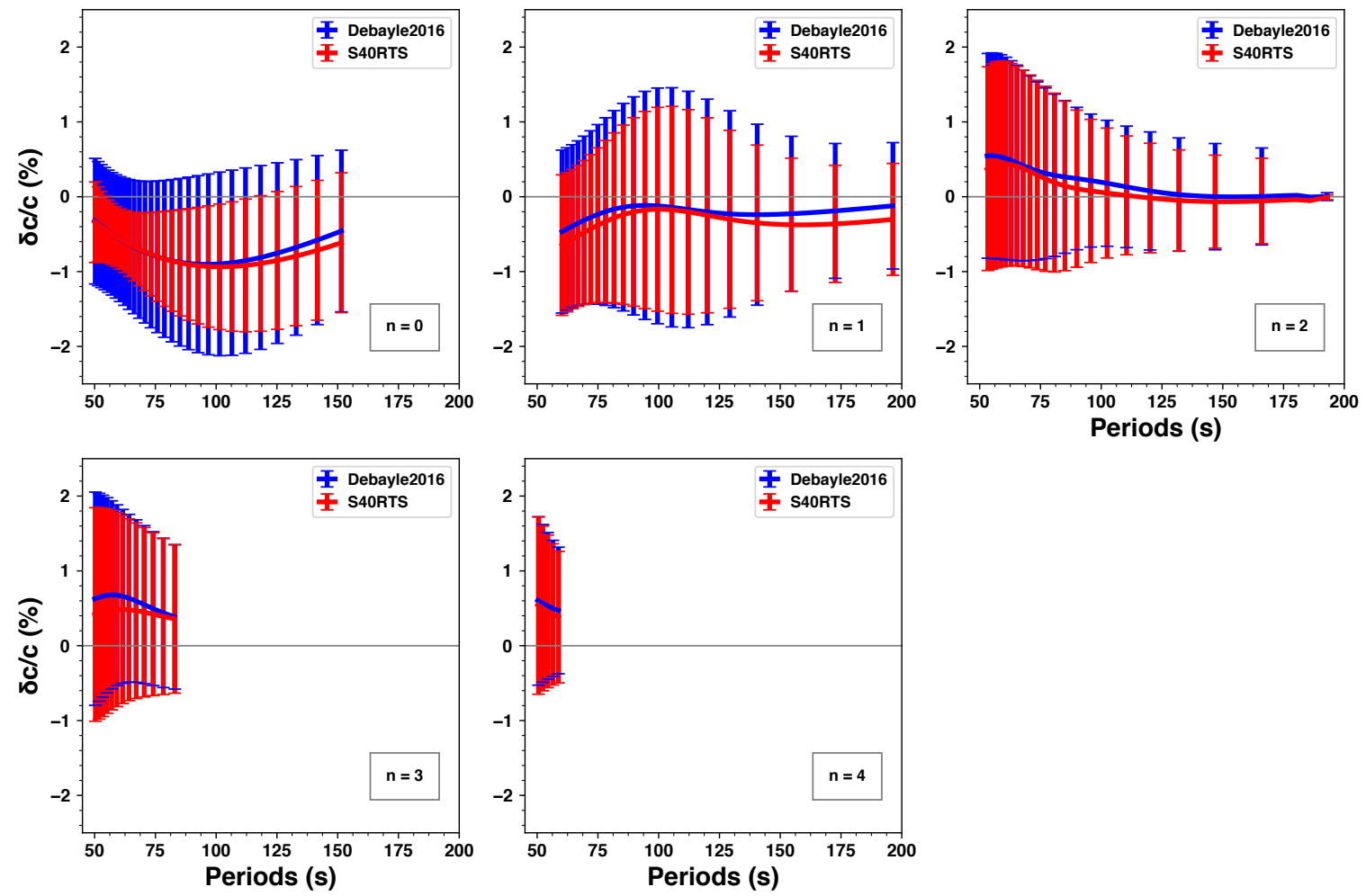

Figure 21. Reliable measured phase velocities expressed as perturbations with respect to PREM for the eastern Australia path and the modes and periods that were estimated to be reliable (Fig. 20). The red curve represents measurements performed using the model of Debayle et al. (2016) as a reference model. The blue curve are measurements performed using model S40RTS of Ritsema et al. (2011) as reference. The phase velocities were converted from perturbations with respect to the 3-D reference model into perturbations with respect to PREM. Uncertainties correspond to $2 \sigma$. 

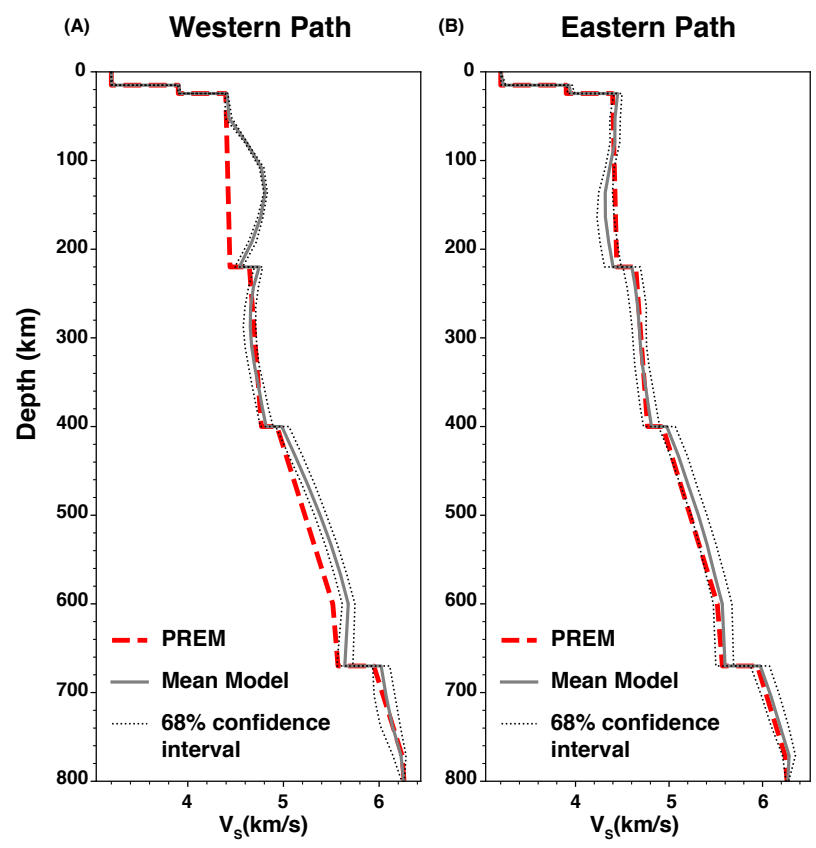

Figure 22. The mean $V_{S}$ solution and one standard deviation for the western (A) and Eastern (B) Australia paths compared to PREM. 